\title{
A transcendental dynamical degree
}

\author{
by \\ JASON P. BELL \\ University of Waterloo \\ Waterloo, ON, Canada \\ JEFFREY DiLleR \\ University of Notre Dame \\ Notre Dame, IN, U.S.A.
}

\section{MATtias Jonsson \\ University of Michigan Ann Arbor, MI, U.S.A.}

\section{Introduction}

The most fundamental dynamical invariant of a dominant rational self-map $f: X \rightarrow X$ of a smooth projective variety is, arguably, its (first) dynamical degree $\lambda(f)$. It can be defined, using intersection numbers, as

$$
\lim _{n \rightarrow \infty}\left(f^{n *} H \cdot H^{\operatorname{dim} X-1}\right)^{1 / n},
$$

where $H$ is any ample divisor. The limit does not depend on the choice of $H$, and it is invariant under birational conjugacy: if $h: X^{\prime}-\rightarrow X$ is a birational map, then

$$
f^{\prime}:=h^{-1} \circ f \circ h: X^{\prime} \rightarrow X^{\prime}
$$

is a dominant rational map with $\lambda\left(f^{\prime}\right)=\lambda(f)$.

The dynamical degree is often difficult to compute. If $f$ is algebraically stable in the sense that $f^{n *}=f^{* n}$ for the induced pullbacks of divisors on $X$ [Sib], then $\lambda(f)$ is equal to the spectral radius of the $\mathbb{Z}$-linear operator $f^{*}: \mathrm{NS}_{\mathbb{R}}(X) \rightarrow \mathrm{NS}_{\mathbb{R}}(X)$ on the real Néron-Severi group $\mathrm{NS}_{\mathbb{R}}(X):=\mathrm{NS}(X) \otimes_{\mathbb{Z}} \mathbb{R}$; hence $\lambda(f)$ is an algebraic integer in that case. For certain classes of maps, such as birational maps of $\mathbb{P}^{2}[\mathrm{DF}]$ or polynomial maps of $\mathbb{A}^{2}$ [FJ1], [FJ2], we can achieve algebraic stability after birational conjugation; hence the dynamical degree is an algebraic integer in these cases. It has been shown, moreover, that the set of dynamical degrees of all rational maps (algebraically stable or not, and over all fields) is countable [BF], [Ur].

All of this leads naturally to the question [BIJ+, Conjecture 13.17]: is the dynamical degree always an algebraic integer, or at least an algebraic number? Surprisingly, the answer is negative. 
MAIn THEOREM. Let $\mathbb{k}$ be a field with char $(\mathbb{k}) \neq 2$. Then, there exists a dominant rational map $f: \mathbb{P}_{\mathbb{k}}^{2--\rightarrow} \mathbb{P}_{\mathbb{k}}^{2}$ whose dynamical degree is a transcendental number.

Our examples are completely explicit, of the form $f=g \circ h$, where

$$
g\left(y_{1}, y_{2}\right)=\left(-y_{1} \frac{1-y_{1}+y_{2}}{1-y_{1}-y_{2}},-y_{2} \frac{1+y_{1}-y_{2}}{1-y_{1}-y_{2}}\right)
$$

is a fixed birational involution, conjugate by a projective linear map to the standard Cremona involution $\left(y_{1}, y_{2}\right) \mapsto\left(y_{1}^{-1}, y_{2}^{-1}\right)$, and

$$
h\left(y_{1}, y_{2}\right)=\left(y_{1}^{a} y_{2}^{b}, y_{1}^{-b} y_{2}^{a}\right)
$$

is a monomial map. We show that, if $(a+b i)^{n} \notin \mathbb{R}$ for all integers $n>0$, then $\lambda(f)$ is transcendental. Favre [Fa] showed that, under the same condition on $a+b i$, the monomial map $h$ cannot be birationally conjugated to an algebraically stable map, though $\lambda(h)=$ $|a+b i|$ is still just a quadratic integer. Rational surface maps, such as $f$, that preserve a rational 2-form were considered as a class by the second author and J.-L. Lin in [DL] (see also [Bla]), where it was shown that failure of stabilizability for $h$ implies the same for $f$. Note that the restriction $\operatorname{char}(\mathbb{k}) \neq 2$ is needed only to ensure that $g$ is non-trivial.

\section{Strategy of the proof}

Our first step toward showing that $\lambda(f)$ is transcendental is to relate degrees of iterates of $h$ to those of $f$. Writing $d_{j}:=\operatorname{deg} h^{j}:=\left(h^{j *} H \cdot H\right)$ for $j \geqslant 0$, with $H \subset \mathbb{P}^{2}$ being a line, we show in $\S 1$ and $\S 2$ that the dynamical degree $\lambda=\lambda(f)$ is the unique positive solution to the equation

$$
\sum_{j=1}^{\infty} d_{j} \lambda^{-j}=1
$$

In order to derive $(\star)$, it is useful to consider the lift of $f$ to various blowups of $\mathbb{P}^{2}$. We use the language of b-divisors to coordinate information about divisors in different blowups. These transform naturally and functorially under the maps $h$ and $g$, so the additional terminology is convenient for understanding the degree growth of $f$; see [FJ1], [BFJ], [Can], [FJ2]. Here, we make use of the additional fact that $h$ and $g$ interact well with the toric structure of $\mathbb{P}^{2}$. This is of course clear for the monomial map $h$, but less evident for the involution $g$.

One computes by elementary means that $d_{j}=\operatorname{Re}\left(\gamma(j) \zeta^{j}\right)$, where $\zeta=a+b i$ and

$$
\gamma(j) \in\{-2, \pm 2 i, 1 \pm 2 i\}
$$


is chosen to be whichever element maximizes the right side. The condition $\zeta^{n} \notin \mathbb{R}$ means that the argument of $\zeta$ is

$$
\operatorname{Arg}(a+b i)=2 \pi \theta
$$

for $\theta \in(0,1)$ irrational. Were $\theta$ rational, the Gaussian integer $\gamma(j)$ would be periodic in $j$, the analytic function

$$
\Delta_{h}(z):=\sum_{j \geqslant 1} d_{j} z^{j}
$$

rational, and $\lambda$ algebraic. However, as Hasselblatt-Propp [HP] observed, when $\theta$ is irrational, the sequence $\left(d_{j}\right)_{j \geqslant 1}$ does not satisfy any linear recurrence relation.

One therefore suspects that $\Delta_{h}(\alpha)$ is unlikely to be algebraic for any given algebraic number $\alpha \neq 0$ in the domain of convergence for the series; in particular, $\Delta_{h}(1 / \lambda)=1$ should force $\lambda$ to be transcendental. There are many results of this type in the literature, see e.g. $[\mathrm{N}],[\mathrm{FM}],[\mathrm{AC} 1],[\mathrm{AC} 2],[\mathrm{Beu}],[\mathrm{AB} 1],[\mathrm{AB} 2],[\mathrm{BBC}]$, but we were not able to locate one that implies directly that at least one of $\lambda$ and $\Delta_{h}\left(\lambda^{-1}\right)$ must be transcendental. Instead, we present in $\S 3$ a proof based on results by Evertse and others on $S$-unit equations; see [EG]. These in turn rely on the $p$-adic subspace theorem by Schlickewei [Sch]. Our method draws inspiration from earlier work of Corvaja and Zannier [CZ] and Adamczewski and Bugeaud [AB1], [AB2], who used the subspace theorem to establish transcendence of special values of certain classes of power series.

The idea is that, if $m / n$ is a continued fraction approximant of $\theta$, then $\zeta^{n}$ is nearly real, the Gaussian integers $\gamma(j)$ are nearly $n$-periodic in $j$, and $\Delta_{h}(z)$ is well approximated by the rational function

$$
\Delta_{h}^{(n)}(z)=\left(1-z^{n}\right)^{-1} \sum_{j=1}^{n} d_{j} z^{j}
$$

obtained by assuming the $\gamma(j)$ are precisely $n$-periodic. If the approximations improve sufficiently quickly with $n$ and $\alpha$ is algebraic, then $\Delta_{h}^{(n)}(\alpha)$ approximates $\Delta_{h}(\alpha)$ too well for the latter to also be algebraic. Unfortunately, this seems a little too much to hope for, without knowing more about how well $\theta$ agrees with its approximants.

To deal with the possibility that $\theta$ is badly approximable by rational numbers, we need a more subtle argument, which uses another result on unit equations, this time by Evertse, Schlickewei and Schmidt [ESS]. In addition, Evertse's theorem on $S$-unit equations does not apply to the rational functions $\Delta_{n}(z)$, and instead we work with related but slightly more complicated functions; see $\S 3$ for details. 


\section{Context}

Dynamical degrees play a key role in algebraic, complex and arithmetic dynamics. With any dominant rational map $f: X \rightarrow X$ of a projective variety $X$ over $\mathbb{k}$ is in fact associated a sequence $\left(\lambda_{p}(f)\right)_{p=1}^{\operatorname{dim} X}$ of dynamical degrees, each invariant under birational conjugation; see [DS1], $[\mathrm{T}],[\mathrm{D}]$. The dynamical degree above corresponds to $p=1$.

Naturally defined in the context of algebraic dynamics, dynamical degrees were first introduced in complex dynamics by Friedland $[\mathrm{Fr}]$, who showed that, when $\mathbb{k}=\mathbb{C}$ and $f$ is a morphism, the topological entropy of $f$ is given by $\log \max _{p} \lambda_{p}(f)$; this generalized earlier work by Gromov, see [Gr], and was later extended (as an inequality) by Dinh and Sibony [DS1] to the case of dominant rational maps. Dynamical degrees are furthermore essential for defining and analyzing natural invariant currents and measures; see for example [RS], [Gu2], [DS2] and the references therein. Their importance from the point of view of complexity and integrability has also been exhibited in the physics literature by Bellon, Viallet and others; see e.g. [BV], [V].

In dimension 2 , the only relevant degrees are $\lambda_{1}=\lambda$ and $\lambda_{2}$ (the 'topological degree', equal to the number of preimages of a typical point, if $\mathbb{k}$ is algebraically closed of characteristic zero). When $\mathbb{k}=\mathbb{C}$, their relationship determines which of two types of dynamical behavior (saddle or repelling) predominates (see [DDG1]-[DDG3] and [Gu1]). The class of examples we consider here includes both types. If, for instance, $\zeta=1+2 i$, then we obtain a map $f$ of small topological degree

$$
\lambda_{2}(f)=\lambda_{2}(h)=|\zeta|^{2}=5<\lambda_{1}(f)=6.8575574092 \ldots
$$

as computed numerically from equation $(\star)$. Replacing $\zeta$ by $\zeta^{2}=-3+4 i$, gives a map with large topological degree

$$
\lambda_{2}(f)=25>\lambda_{1}(f)=13.4496076817 \ldots
$$

In arithmetic dynamics, $\mathbb{k}$ is a global field, and the (first) dynamical degree serves as an upper bound for the asymptotics of the growth of heights along orbits [Sil], [KS], [Mat]; the question of when equality holds is part of the Kawaguchi-Silverman conjecture, which recently has attracted a lot of attention.

\section{Outlook}

As already mentioned, the set of all possible dynamical degrees is countable, and our main theorem shows that it contains transcendental numbers. It would obviously be interesting to say more about it. Note that the set of dynamical degrees of birational surface maps 
is much better understood; see e.g. [BK1], $[\mathrm{McM}],[\mathrm{Ue}],[\mathrm{BC}]$. It would be interesting to know (see e.g. [V, p.1379]) if a birational map $f: \mathbb{P}^{k} \rightarrow \mathbb{P}^{k}$ can have transcendental dynamical degree when $k \geqslant 3$. We intend to address this in a future article, though the number theoretic details seem more complicated. See $[C X],[D F]$ for some other results about degree growth of rational maps in higher dimensions.

It would also be interesting to study the complex and arithmetic dynamics of the rational map $f=f_{\zeta}$ considered here. For example, does $f$ admit a unique measure of maximal entropy, and is the topological entropy equal to $\log \lambda(f)$ ? The fact that $f$ is defined over $\mathbb{Q}$ may be useful, see e.g. [JR], where it is shown that (complex) birational surface maps defined over $\overline{\mathbb{Q}}$ always admit a measure of maximal entropy $\log \lambda(f)$. On the arithmetic side, one may ask whether the Kawaguchi-Silverman conjecture holds: does every point with Zariski dense orbit have arithmetic degree equal to $\lambda(f)$ ? Note that what we call the Kawaguchi-Silverman conjecture is part (d) of [KS, Conjecture 6]. Given our main theorem, the existence of a point as above would in fact contradict part (b); see also [LS].

Acknowledgments. We thank J. Blanc, S. Kawaguchi, C. T. McMullen, M. Satriano, U. Zannier and, especially, H. Krieger, for valuable comments. The last two authors thank J.-L. Lin and P. Reschke for their help during an earlier stage of this project, and J. Lagarias and B. Poonen for useful pointers regarding transcendence questions. Finally, we thank the referee for a careful reading and many thoughtful suggestions. The first author was partially supported by NSERC grant RGPIN-2016-03632; the second author by NSF grant DMS-1954335; and the last author by NSF grants DMS-1600011 and DMS-1900025, and the United States-Israel Binational Science Foundation. The final form of the present collaboration originated at the Simons Symposium in Complex, Algebraic, and Arithmetic Dynamical Systems in May 2019; we are very grateful to the Simons Foundation for its generous support.

\section{Dominant rational maps of the projective plane}

In this section we study dominant rational self-maps of $\mathbb{P}^{2}$ using the induced action on b-divisor classes. The exposition largely follows $[\mathrm{BFJ}]$ and $[\mathrm{DL}]\left({ }^{1}\right)$ but with particular attention paid to the structure of $\mathbb{P}^{2}$ as a toric variety. We work over a field $\mathbb{k}$ of characteristic different from 2. Since degrees of rational maps are invariant under ground field extension, we may and will assume that $\mathbb{k}$ is algebraically closed. The assumption that $\operatorname{char} \mathbb{k} \neq 2$ will be used in $\S 2$.

( $\left.{ }^{1}\right)$ Both of these articles were written for surfaces defined over $\mathbb{C}$, but the results we use from them work with proofs unchanged over any algebraically closed field. 


\subsection{Setup}

Fix homogeneous coordinates $\left[x_{0}: x_{1}: x_{2}\right]$ on $\mathbb{P}^{2}$ and use affine coordinates

$$
\left(y_{1}, y_{2}\right)=\left(\frac{x_{1}}{x_{0}}, \frac{x_{2}}{x_{0}}\right)
$$

on the affine chart $\left\{x_{0} \neq 0\right\} \simeq \mathbb{A}^{2}$. Recall that $\mathbb{P}^{2}$ is a toric surface with torus

$$
\mathbb{T}=\mathbb{G}_{m}^{2}=\left\{x_{0} x_{1} x_{2} \neq 0\right\},
$$

and torus-invariant prime divisors being the coordinate lines $\left\{x_{j}=0\right\}, j=0,1,2$.

\subsection{Rational maps and their degrees}

A dominant rational self-map of $\mathbb{P}^{2}$ is given in homogeneous coordinates by

$$
f:\left[x_{0}: x_{1}: x_{2}\right] \longmapsto\left[f_{0}\left(x_{0}, x_{1}, x_{2}\right): f_{1}\left(x_{0}, x_{1}, x_{2}\right): f_{2}\left(x_{0}, x_{1}, x_{2}\right)\right],
$$

where $f_{0}, f_{1}$ and $f_{2}$ are homogeneous polynomials of the same degree $d \geqslant 1$, and with no factor in common. The integer $\operatorname{deg} f:=d$ is called the degree of $f$; see also equation (1.4).

The sequence $\left(\operatorname{deg} f^{n}\right)_{n \geqslant 1}$ is submultiplicative, i.e.

$$
\operatorname{deg} f^{m+n} \leqslant \operatorname{deg} f^{m} \cdot \operatorname{deg} f^{n}
$$

hence, the limit

$$
\lambda(f)=\lim _{n \rightarrow \infty}\left(\operatorname{deg} f^{n}\right)^{1 / n}=\inf _{n}\left(\operatorname{deg} f^{n}\right)^{1 / n} \in[1, \infty)
$$

exists and is equal to the dynamical degree of $f$ as defined in the introduction.

\subsection{Monomial maps}

Any $2 \times 2$ matrix $\Lambda=\left(a_{j k}\right)_{j, k}$ with integer coefficients and non-zero determinant defines a dominant rational self-map $h_{\Lambda}: \mathbb{P}^{2} \rightarrow \mathbb{P}^{2}$, which in affine coordinates is given by

$$
h_{\Lambda}:\left(y_{1}, y_{2}\right) \longmapsto\left(y_{1}^{a_{11}} y_{2}^{a_{12}}, y_{1}^{a_{21}} y_{2}^{a_{22}}\right) .
$$

Such rational maps are called monomial maps; they correspond to surjective endomorphisms of the algebraic group $\mathbb{T}$.

Note that $h_{\Lambda_{1} \Lambda_{2}}=h_{\Lambda_{1}} \circ h_{\Lambda_{2}}$. The degree of a monomial map is given by

$$
\operatorname{deg} h_{\Lambda}=\max \left\{0, a_{11}+a_{12}, a_{21}+a_{22}\right\}+\max \left\{0,-a_{11},-a_{12}\right\}+\max \left\{0,-a_{21},-a_{22}\right\} ;
$$



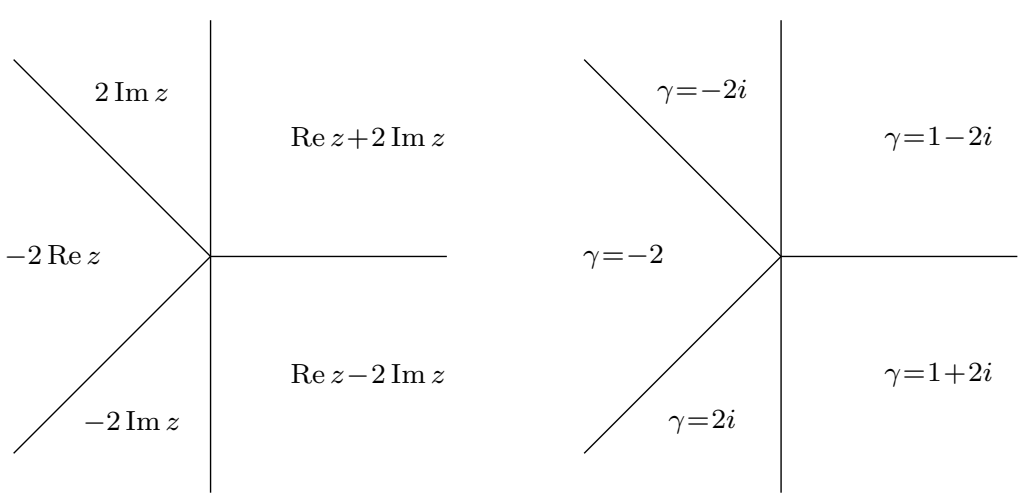

Figure 1. The left picture shows the piecewise $\mathbb{R}$-linear function $\psi: \mathbb{C} \rightarrow \mathbb{R} \geqslant 0$ defined by equation (1.1). The right picture shows the element $\gamma \in \Gamma_{0}$ that realizes the maximum in the definition of $\psi$. Note that the angles that the rays make with the positive real axis are all integer multiples of $\frac{1}{4} \pi$.

see $[\mathrm{HP}],[\mathrm{BK} 2]$.

We can view $\Lambda$ as a linear self-map of $\mathbb{Z}^{2}$ or $\mathbb{R}^{2}$. Now, identify $\mathbb{R}^{2}$ with $\mathbb{C}$ and assume that $\Lambda$ is given by multiplication with a Gaussian integer $\zeta \in \mathbb{Z}[i]$, that is,

$$
\Lambda=\Lambda_{\zeta}=\left(\begin{array}{cc}
\operatorname{Re} \zeta & -\operatorname{Im} \zeta \\
\operatorname{Im} \zeta & \operatorname{Re} \zeta
\end{array}\right)
$$

In this case, we write $h_{\zeta}=h_{\Lambda_{\zeta}}$. Note that $h_{\zeta_{1}} \circ h_{\zeta_{2}}=h_{\zeta_{1} \zeta_{2}}$. We have

$$
\operatorname{deg} h_{\zeta}=\max \{0, \operatorname{Re} \zeta-\operatorname{Im} \zeta, \operatorname{Re} \zeta+\operatorname{Im} \zeta\}+\max \{0,-\operatorname{Re} \zeta, \operatorname{Im} \zeta\}+\max \{0,-\operatorname{Re} \zeta,-\operatorname{Im} \zeta\} .
$$

which we can rewrite as $\operatorname{deg} h_{\zeta}=\psi(\zeta)$, where $\psi: \mathbb{C} \rightarrow \mathbb{R}_{\geqslant 0}$ is a convex piecewise $\mathbb{R}$-linear function given by

$$
\psi(z):=\max _{\gamma \in \Gamma_{0}} \operatorname{Re}(\gamma z), \quad \text { where } \Gamma_{0}:=\{-2, \pm 2 i, 1 \pm 2 i\} ;
$$

see Figure 1.

One checks that $\psi$ is comparable to the Euclidean norm on $\mathbb{C}$; specifically,

$$
|z| \leqslant \psi(z) \leqslant \sqrt{5}|z| .
$$

Since $h_{\zeta}^{n}=h_{\zeta^{n}}$ for $n \geqslant 1$, it follows that the dynamical degree of $h_{\zeta}$ is

$$
\lim _{n \rightarrow \infty} \psi\left(\zeta^{n}\right)^{1 / n}=|\zeta| .
$$

We will be interested in the case when $\zeta^{n} \notin \mathbb{R}$ for all $n \geqslant 1$. This is equivalent to $\zeta$ not being an integer multiple of $1, i$ or $1 \pm i$; see e.g. [Cal, main lemma]. In this case, there is, for every $n \geqslant 1$, a unique element $\gamma(n) \in \Gamma_{0}$ such that $\psi\left(\zeta^{n}\right)=\operatorname{Re}\left(\gamma(n) \zeta^{n}\right)$. 


\subsection{Blowups}

By a blowup of $\mathbb{P}^{2}$ we mean a birational morphism $\pi: X_{\pi} \rightarrow \mathbb{P}^{2}$, where $X_{\pi}$ is a smooth projective surface. Up to isomorphism, $\pi$ is then a finite composition of point blowups [Sha, Theorem 4.10]. If $\pi$ and $\pi^{\prime}$ are blowups of $\mathbb{P}^{2}$, then

$$
\mu:=\pi^{-1} \circ \pi^{\prime}: X_{\pi^{\prime}--\rightarrow} X_{\pi}
$$

is a birational map; we say that $\pi^{\prime}$ dominates $\pi$, written $\pi^{\prime} \geqslant \pi$, if $\mu$ is a morphism. Any two blowups can be dominated by a third one, as follows by applying [Sha, Theorem 4.9] to the birational map $\mu$ above. It follows that the set $\mathfrak{B l}$ of isomorphism classes of blowups is a directed set.

\subsection{Primes over the projective plane}

We will say that prime divisors $E \subset X_{\pi}$ and $E^{\prime} \subset X_{\pi^{\prime}}$ in different blowups are equivalent if there is a blowup $\pi^{\prime \prime}=\pi \circ \mu=\pi^{\prime} \circ \mu^{\prime}$ dominating both $\pi$ and $\pi^{\prime}$, and a prime divisor $E^{\prime \prime} \subset X_{\pi^{\prime \prime}}$ such that $E=\mu\left(E^{\prime \prime}\right)$ and $E^{\prime}=\mu^{\prime}\left(E^{\prime \prime}\right)$. We let $\mathcal{P}$ denote the set of all the resulting equivalence classes and call each $E \in \mathcal{P}$ a prime over $\mathbb{P}^{2}$.

We say that a blowup $\pi$ expresses a prime $E \in \mathcal{P}$, if $E$ is represented by a prime divisor in $X_{\pi}$ that we call then the center of $E$ on $X_{\pi}$. Slightly abusively, we use the same letter to denote the center, writing $E \subset X_{\pi}$.

If a blowup $\pi$ does not express a prime $E$, then we can choose a further blowup $\pi^{\prime}=\pi \circ \mu>\pi$ such that $E$ is represented by a prime divisor on $X_{\pi^{\prime}}$. The image under $\mu$ of this prime divisor is a point in $X_{\pi}$ which does not depend on the choice of $\pi^{\prime}$ and which we call the center of $E$ on $X_{\pi}$.

\subsection{The group of b-divisors}

For any blowup $\pi$ of $\mathbb{P}^{2}$, denote by $\operatorname{Pic}\left(X_{\pi}\right)$ the Picard group on $X_{\pi}$, i.e. the set of linear equivalence classes of (Cartier) divisors on $X_{\pi}$. When $\pi^{\prime} \geqslant \pi$, the birational morphism $\mu: X_{\pi^{\prime}} \rightarrow X_{\pi}$ induces an injective homomorphism $\mu^{*}: \operatorname{Pic}\left(X_{\pi}\right) \rightarrow \operatorname{Pic}\left(X_{\pi^{\prime}}\right)$. The group of $b$-divisor classes on $\mathbb{P}^{2}$ is defined as the direct limit

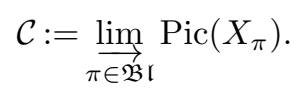

Concretely, an element of $\mathcal{C}$ is an element of $\operatorname{Pic}\left(X_{\pi}\right)$ for some blowup $\pi$, where two elements $A \in \operatorname{Pic}\left(X_{\pi}\right)$ and $A^{\prime} \in \operatorname{Pic}\left(X_{\pi^{\prime}}\right)$ are identified if and only if they pull back to 
the same class on some blowup dominating both $\pi$ and $\pi^{\prime}$. A class in the image of $\operatorname{Pic}\left(X_{\pi}\right) \hookrightarrow \mathcal{C}$ is said to be determined on $X_{\pi}$. We let

$$
H=\mathcal{O}_{\mathbb{P}^{2}}(1) \in \mathcal{C}
$$

denote the class determined by a line in $\mathbb{P}^{2}$.

Remark 1.1. The 'b' in 'b-divisor' stands for birational, following Shokurov. In [BFJ], the elements of $\mathcal{C}$ were referred to as Cartier classes on the Riemann-Zariski space of $\mathbb{P}^{2}$. The space $\mathcal{C}$ appears earlier in [Man], where it is denoted $Z \cdot\left(\mathbb{P}^{2}\right)$. Note that since each surface $X_{\pi}$ is rational, the Picard group $\operatorname{Pic}\left(X_{\pi}\right)$ coincides with the Néron-Severi group $\operatorname{NS}\left(X_{\pi}\right)$.

There is a natural intersection pairing $\mathcal{C} \times \mathcal{C} \rightarrow \mathbb{Z}$, denoted $(A \cdot B)$ for $A, B \in \mathcal{C}$. This is defined as the intersection number on any blowup $X_{\pi}$, where $A$ and $B$ are both determined (see [BFJ, §1.4] or [Man, §34.7]).

\subsection{Toric blowups}

We call a blowup $\pi: X_{\pi} \rightarrow \mathbb{P}^{2}$ toric if $X_{\pi}$ is also a toric surface and $\pi$ is equivariant with respect to the torus action. Concretely, $\pi=\pi_{1} \circ \ldots \circ \pi_{n}$, where each factor $\pi_{j}: X_{j} \rightarrow X_{j-1}$ is a point blowup centered at the intersection of two different torus-invariant prime divisors in $X_{j-1}$.

If $\pi$ is a toric blowup of $\mathbb{P}^{2}$ and $E \subset X_{\pi}$ a torus-invariant prime divisor, then a point $p \in E$ is called free if it does not belong to any other torus-invariant prime divisor on $X_{\pi}$, i.e. its orbit under the torus action is 1-dimensional.

We will call $E \in \mathcal{P}$ a toric prime if there is a toric blowup $\pi$ that expresses $E$ as a torus-invariant prime divisor. Let $\mathcal{P}^{\text {tor }}$ denote the set of all toric primes.

Proposition 1.2. Any blowup $\pi$ of $\mathbb{P}^{2}$ factors uniquely as $\pi=\pi_{\text {tor }} \circ \mu$ into a toric blowup $\pi_{\text {tor }}$ that expresses the same set of toric primes as $\pi$ and a birational morphism $\mu: X_{\pi} \rightarrow X_{\pi_{\mathrm{tor}}}$ that contracts only non-toric primes.

Proof. This follows from [DL, Corollary 5.5] and the fact that the toric primes in $X_{\pi}$ are precisely the (simple) poles of the rational 2 -form

$$
\pi^{*} \frac{d y_{1} \wedge d y_{2}}{y_{1} y_{2}} .
$$

With each prime $E \in \mathcal{P}$ we associate an order of vanishing valuation

$$
\operatorname{ord}_{E}: \mathbb{k}\left(\mathbb{P}^{2}\right)^{\times} \longrightarrow \mathbb{Z}
$$


by choosing a blowup $\pi$ such that $E \subset X_{\pi}$, and setting $\operatorname{ord}_{E}(\varphi)$ equal to the coefficient of $E$ in the divisor of the rational function $\varphi \circ \pi$ on $X_{\pi}$. We define a 'tropicalization' map trop: $\mathcal{P} \rightarrow \mathbb{Z}^{2}$ by

$$
\operatorname{trop}(E)=\left(\operatorname{ord}_{E}\left(y_{1}\right), \operatorname{ord}_{E}\left(y_{2}\right)\right),
$$

where $\left(y_{1}, y_{2}\right)$ are the affine coordinates fixed above. Note that $\operatorname{trop}(E)=(0,0)$ for all non-toric primes $E \subset \mathbb{P}^{2}$, whereas if we write $H_{j}:=\left\{x_{j}=0\right\}, j=0,1,2$, then

$$
\operatorname{trop}\left(H_{0}\right)=(-1,-1), \quad \operatorname{trop}\left(H_{1}\right)=(1,0) \quad \text { and } \quad \operatorname{trop}\left(H_{2}\right)=(0,1) .
$$

In any blowup $\pi$ of $\mathbb{P}^{2}$, the divisors of the rational functions $y_{j} \circ \pi, j=1,2$, have simple normal crossings support. Hence, if $\mu: X_{\pi^{\prime}} \rightarrow X_{\pi}$ is the point blowup at $p \in X_{\pi}$, then the prime $E^{\prime}$ contracted by $\mu$ satisfies

$$
\operatorname{trop}\left(E^{\prime}\right)=\sum_{E \subset X_{\pi}: p \in E} \operatorname{trop}(E)
$$

We call a non-zero element $t \in \mathbb{Z}^{2}$ primitive if $t \notin m \mathbb{Z}^{2}$ for any integer $m \geqslant 2$. The next result follows easily by induction from the discussion above, and is related to the fact that $\mathrm{SL}_{2}(\mathbb{Z})$ acts transitively on primitive elements of $\mathbb{Z}^{2}$.

Proposition 1.3. The map trop restricts to a bijection from $\mathcal{P}^{\text {tor }}$ onto the set of primitive elements $t \in \mathbb{Z}^{2}$.

We will say that elements $s, t \in \mathbb{Z}^{2}$ are commensurate if $s=r t$ for some positive $r \in \mathbb{Q}$. For each non-zero (but not necessarily primitive) element $t \in \mathbb{Z}^{2}$, we let $E_{t} \in \mathcal{P}^{\text {tor }}$ be the unique toric prime such that $\operatorname{trop}\left(E_{t}\right)$ is commensurate with $t$.

Proposition 1.4. Let $\pi=\pi_{\text {tor }}{ }^{\circ} \mu$ be a blowup of $\mathbb{P}^{2}$, factored as in Proposition 1.2, and $E \subset X_{\pi}$ be a non-toric prime divisor with $t:=\operatorname{trop}(E) \neq(0,0)$. Then, the following statements hold:

(i) $\pi_{\text {tor }}$ expresses the toric prime $E_{t}$, and $\mu(E)$ is a free point on $E_{t}$;

(ii) if $E^{\prime} \in \mathcal{P}$ is not expressed in $X_{\pi}$, and its center on $X_{\pi}$ is a point $p^{\prime} \in E$, then $E^{\prime}$ is also non-toric, and $\operatorname{trop}\left(E^{\prime}\right)$ is commensurate with $t$.

Proof. The support of the divisor of the rational function $y_{j} \circ \pi_{\text {tor }}$ on $X_{\pi_{\text {tor }}}$ does not meet the torus $\mathbb{T}$, for $j=1,2$, so since $E \notin \mathcal{P}^{\text {tor }}$ and $\operatorname{trop}(E) \neq(0,0)$, we have that $\mu(E)$ is a point in $X_{\pi_{\text {tor }}} \backslash \mathbb{T}$. If $p$ is the intersection of two distinct toric primes expressed by $\pi_{\text {tor }}$, then $\pi$ dominates $\pi_{\text {tor }} \circ \mu^{\prime}$, where $\mu^{\prime}: X_{\pi^{\prime}} \rightarrow X_{\pi_{\text {tor }}}$ is the point blowup at $p$. This means, however, that $\mu^{\prime-1}(p)$ is a toric prime expressed by $\pi$ but not $\pi_{\text {tor }}$, which contradicts the choice of $\pi_{\text {tor }}$. 
Thus, $p$ is a free point on a prime $E_{s}$ expressed by $\pi_{\text {tor }}$, with $s=\operatorname{trop}\left(E_{s}\right)$. The map trop therefore vanishes along all other primes expressed by $\pi_{\text {tor }}$ that contain $p$. Hence, by factoring $\mu$ into point blowups and repeatedly applying equation (1.3), we see that $t=\operatorname{trop}(E)$ is commensurate with $s$. So (i) holds, and we turn to (ii).

By the previous step, any prime that is expressed by $\pi$ and contains $p^{\prime}$ has tropicalization equal to a multiple (possibly zero) of $t$. Hence, we can choose a blowup $\pi^{\prime}=\pi \circ \mu^{\prime}>\pi$ that expresses $E^{\prime}$, factor $\mu^{\prime}$ into point blowups and repeatedly apply equation (1.3) to obtain that $\operatorname{trop}\left(E^{\prime}\right)$ is commensurate with $t$. Since $E^{\prime} \neq E_{t}$, Proposition 1.3 tells us that $E^{\prime}$ is not toric.

The set of toric b-divisor classes $\mathcal{C}^{\text {tor }} \subset \mathcal{C}$ is the direct limit

$$
\underset{\pi}{\lim _{\pi}} \operatorname{Pic}\left(X_{\pi}\right)
$$

where $\pi$ runs over all toric blowups of $\mathbb{P}^{2}$. Each class in $\operatorname{Pic}\left(X_{\pi}\right)$ is represented by a toric divisor, i.e. a divisor with support equal to a collection of toric primes expressed by $\pi$. In particular $H \in \mathcal{C}^{\text {tor }}$, and a class in $\mathcal{C}^{\text {tor }}$ is orthogonal to $H$ if and only if it is represented by a $\pi$-exceptional toric divisor on some toric blowup $\pi$ of $X$. We will use this fact below in proving Lemma 2.6.

\subsection{Action by rational maps on primes and on b-divisor classes}

Consider a dominant rational map $f: \mathbb{P}^{2} \rightarrow \rightarrow \mathbb{P}^{2}$. For any blowups $\pi$ and $\pi^{\prime}$ of $\mathbb{P}^{2}$ we have an induced rational map $f_{\pi \pi^{\prime}}:=\pi^{-1} \circ f \circ \pi^{\prime}: X_{\pi^{\prime} \rightarrow-} X_{\pi}$. Given $\pi$, we can choose $\pi^{\prime}$ such that $f_{\pi \pi^{\prime}}$ is a morphism, as follows from [Sha, Theorem 4.8]. We now define a group homomorphism

$$
f^{*}: \mathcal{C} \longrightarrow \mathcal{C}
$$

as follows: if $A \in \mathcal{C}$ is determined on $X_{\pi}$, pick a blowup $\pi^{\prime}$ such that $f_{\pi \pi^{\prime}}: X_{\pi^{\prime}} \rightarrow X_{\pi}$ is a morphism, and declare $f^{*} A \in \mathcal{C}$ to be the class determined on $X_{\pi^{\prime}}$ by $f_{\pi \pi^{\prime}}^{*} A$. This action is functorial: if $f$ and $g$ are dominant rational maps of $\mathbb{P}^{2}$, then $(f \circ g)^{*}=g^{*} f^{*}$ on $\mathcal{C}$. When $h: \mathbb{P}^{2} \rightarrow \rightarrow \mathbb{P}^{2}$ is monomial, we have $h^{*} \mathcal{C}^{\text {tor }} \subset \mathcal{C}^{\text {tor }}$. The degree of a rational map can be computed as follows:

$$
\operatorname{deg} f=\left(f^{*} H \cdot H\right) .
$$

The rational map $f$ also induces an action $f: \mathcal{P} \rightarrow \mathcal{P}$ on the set of all primes over $\mathbb{P}^{2}$. If $\pi^{\prime}$ is a blowup expressing $E \in \mathcal{P}$, then as in [BFJ] (see just before Lemma 2.4) there exists another blowup $\pi$ such that the lift $f_{\pi \pi^{\prime}}: X_{\pi^{\prime}-\rightarrow} X_{\pi}$ does not contract any curves. We set

$$
f(E):=f_{\pi \pi^{\prime}}(E)
$$


Proposition 1.5. For any monomial map $h: \mathbb{P}^{2} \rightarrow \rightarrow \mathbb{P}^{2}$, with associated matrix $\Lambda$, and any prime $E \in \mathcal{P}$, the following statements hold:

(i) $h(E)$ is toric if and only if $E$ is;

(ii) $\operatorname{trop}(h(E))$ is commensurate with $\Lambda(\operatorname{trop}(E))$.

Proof. The first conclusion follows from the first conclusion of [DL, Corollary 6.3] and the fact that

$$
h^{*} \frac{d y_{1} \wedge d y_{2}}{y_{1} y_{2}}=(\operatorname{det} \Lambda) \frac{d y_{1} \wedge d y_{2}}{y_{1} y_{2}} .
$$

The second conclusion is a (by now) standard computation.

\section{The degree sequence of certain rational maps}

We now specialize the considerations above to a particular class of maps that will later be shown to have transcendental dynamical degrees.

\subsection{A volume-preserving involution}

As in [DL], we consider the involution $\left(^{2}\right) g: \mathbb{P}^{2} \rightarrow \rightarrow \mathbb{P}^{2}$ defined in homogeneous coordinates by

$$
g:\left[x_{0}: x_{1}: x_{2}\right] \longmapsto\left[x_{0}\left(x_{1}+x_{2}-x_{0}\right): x_{1}\left(x_{2}+x_{0}-x_{1}\right): x_{2}\left(x_{0}+x_{1}-x_{2}\right)\right] .
$$

In affine coordinates

$$
\left(y_{1}, y_{2}\right)=\left(\frac{x_{1}}{x_{0}}, \frac{x_{2}}{x_{0}}\right)
$$

this becomes

$$
g:\left(y_{1}, y_{2}\right) \longmapsto\left(-y_{1} \frac{1-y_{1}+y_{2}}{1-y_{1}-y_{2}},-y_{2} \frac{1+y_{1}-y_{2}}{1-y_{1}-y_{2}}\right) .
$$

The projective linear automorphism

$$
A:\left[x_{0}: x_{1}: x_{2}\right] \longmapsto\left[x_{1}+x_{2}-x_{0}: x_{2}+x_{0}-x_{1}: x_{0}+x_{1}-x_{2}\right]
$$

conjugates $g$ to the Cremona involution

$$
A g A^{-1}:\left[x_{0}: x_{1}: x_{2}\right] \longmapsto\left[x_{1} x_{2}: x_{2} x_{0}: x_{0} x_{1}\right]
$$

As a consequence, we have the following geometric description. Consider the three points $p_{0}=[0: 1: 1], p_{1}=[1: 0: 1]$ and $p_{2}=[1: 1: 0]$, and the three lines

$$
L_{0}=\left\{x_{0}=x_{1}+x_{2}\right\}, \quad L_{1}=\left\{x_{1}=x_{2}+x_{0}\right\} \quad \text { and } \quad L_{2}=\left\{x_{2}=x_{0}+x_{1}\right\}
$$

$\left({ }^{2}\right)$ Here, we use that the ground field has characteristic different from 2. Indeed, $g$ is the identity in characteristic 2 . 


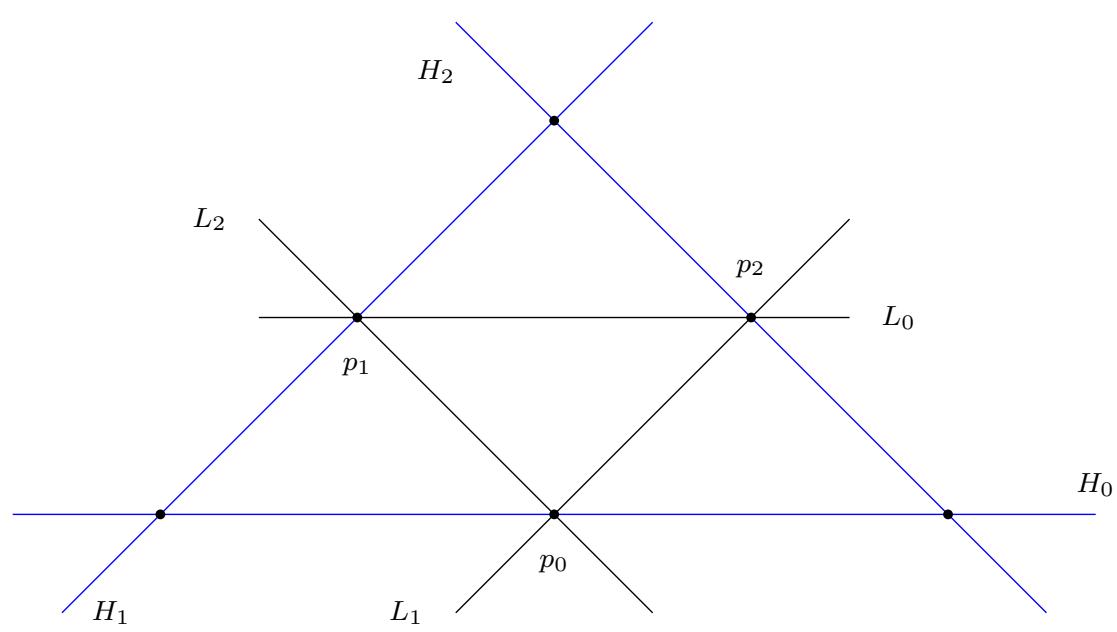

Figure 2. The birational involution $g$ contracts the line $L_{j}$ to the point $p_{j}, j=0,1,2$. It leaves the coordinate lines $H_{j}=\left\{x_{j}=0\right\}$ invariant. The restriction $\left.g\right|_{H_{j}}$ fixes the two points $H_{j} \cap H_{l}$, $l \neq j$, and sends $p_{j}$ to the point $H_{j} \cap L_{j}$ (which is not shown).

on $\mathbb{P}^{2}$. Let $X^{0}$ be the blowup of $\mathbb{P}^{2}$ at $\left\{p_{0}, p_{1}, p_{2}\right\}$, with exceptional divisors $F_{0}, F_{1}$ and $F_{2}$. Then, $g$ induces an automorphism of $X^{0}$ of order 2 that sends $F_{j}$ to the strict transform of $L_{j}$, for $j=0,1,2$.

Let $\pi: X_{\pi} \rightarrow \mathcal{P}^{2}$ be a toric blowup. For $j=1,2,3$, the point $p_{j} \in \mathbb{P}^{2}$ is a free point on the toric prime $H_{j}$. Hence, its preimage by $\pi$ remains a free point on $H_{j}$, and we continue to denote it by $p_{j}$. We let $\pi^{0}: X_{\pi}^{0} \rightarrow \mathbb{P}^{2}$ be the blowup of $X_{\pi}$ along the set $\left\{p_{0}, p_{1}, p_{2}\right\} \subset X_{\pi}$.

Lemma 2.1. For any toric blowup $\pi: X_{\pi} \rightarrow \mathbb{P}^{2}$, the induced birational map

$$
g_{\pi \pi}^{0}: X_{\pi}^{0} \rightarrow X_{\pi}^{0}
$$

is a morphism that fixes each toric prime $E \subset X^{0}$.

Proof. We have already explained that this is true when $X_{\pi}=\mathbb{P}^{2}$. Hence, it suffices by induction to show that if the lemma holds for some toric blowup $\pi$, then it also holds for the toric blowup $\pi^{\prime}=\pi \circ \mu$, where $\mu$ is the point blowup of the intersection of two toric primes $E_{s}, E_{t} \subset X_{\pi}$. But the facts that $g$ fixes both $E_{s}$ and $E_{t}$, and that $E_{s} \cap E_{t}$ is distinct from $p_{1}, p_{2}$ and $p_{3}$ imply that $E_{s} \cap E_{t}$ remains a point in $X_{\pi}^{0}$, and that the automorphism $g_{\pi \pi}^{0}$ fixes it. Hence, $g_{\pi^{\prime} \pi^{\prime}}^{0}$ is an automorphism fixing the exceptional prime

$$
E=\mu^{-1}\left(E_{s} \cap E_{t}\right) .
$$

Lemma 2.2. The induced map $g: \mathcal{P} \rightarrow \mathcal{P}$ is a bijection that fixes the subset $\mathcal{P}^{\text {tor }}$ pointwise. If $E \in \mathcal{P}$ is a prime such that $\operatorname{trop}(E)$ is incommensurate with $(0,0),(-1,-1)$, $(1,0)$ and $(0,1)$, then $\operatorname{trop}(g(E))$ is commensurate with $\operatorname{trop}(E)$. 
Though it is not strictly necessary for the proof, we note the related fact that $D g\left(q_{j k}\right)=-I$ at each fixed point $q_{j k}=H_{j} \cap H_{k}$.

Proof. Set $t=\operatorname{trop}(E)$. The first assertion follows from Lemma 2.1. For the second assertion, we may assume that $E$ is not toric. If $\pi=\pi_{\text {tor }} \circ \mu$ is a blowup that expresses $E$, decomposed as in Proposition 1.2, then Proposition 1.4 tells us that $\mu(E)$ is a free point on the toric prime $E_{t} \subset X_{\pi_{\text {tor }}}$. Since $t$ is incommensurate with $(-1,-1),(1,0)$ and $(0,1)$, we have $\mu(E) \notin\left\{p_{1}, p_{2}, p_{3}\right\}$; see equation (1.2). So, by Lemma 2.1 , the map $g_{\pi_{\text {tor }} \pi_{\text {tor }}}$ is a local isomorphism about $\mu(E)$ and the image $g_{\pi_{\text {tor }} \pi_{\text {tor }}}(\mu(E))$ is also a free point in $E_{t}$. Thus, $g(E)$ is a non-toric prime over a free point in $E_{t}$, and $\operatorname{trop}(g(E))$ is commensurate with $t$.

Now, consider a monomial map $h=h_{\zeta}$ associated (as in $\S 1.3$ ) with a Gaussian integer $\zeta$ for which $\zeta^{n} \notin \mathbb{R}$ for all $n \geqslant 1$. We will construct a set $\mathcal{P}^{\prime} \subset \mathcal{P}$ of primes over $\mathbb{P}^{2}$ that is backward invariant under both $g$ and $h$. As before, we identify $\mathbb{R}^{2}$ with $\mathbb{C}$. Define

$$
N^{\prime}:=\bigcup_{j \geqslant 1} \zeta^{-j} \mathbb{R}_{>0}\{1, i,-1-i\} \subset \mathbb{C} .
$$

Our assumption on $\zeta$ implies that $N^{\prime}$ is an infinite set of rays in $\mathbb{C}$, none of which containing $0,1, i$ or $-1-i$. Let

$$
\mathcal{P}^{\prime}:=\left\{E \in \mathcal{P} \backslash \mathcal{P}^{\text {tor }}: \operatorname{trop}(E) \in N^{\prime}\right\} .
$$

Corollary 2.3. We have $g^{-1}\left(\mathcal{P}^{\prime}\right) \subset \mathcal{P}^{\prime}$ and $h^{-1}\left(\mathcal{P}^{\prime}\right) \subset \mathcal{P}^{\prime}$.

Proof. The first inclusion follows from Lemma 2.2 and the fact that $1, i$ and $-1-i$ are not in $N^{\prime}$. The second one follows from Proposition 1.5 and the fact that the matrix $\Lambda$ associated with the monomial map $h$ acts on $\mathbb{R}^{2} \simeq \mathbb{C}$ by multiplication with $\zeta$.

Next, we study the action of $g$ and $h$ on the group $\mathcal{C}$ of b-divisor classes. Define $\mathcal{C}^{\prime} \subset \mathcal{C}$ to be the subgroup of classes that can be represented by a divisor $D$ on some blowup of $\mathbb{P}^{2}$, such that all irreducible components of $D$ lie in $\mathcal{P}^{\prime}$. Proposition 1.4 implies that $\mathcal{C}^{\prime}$ is orthogonal to $\mathcal{C}^{\text {tor }}$, and in particular to $H=\mathcal{O}_{\mathbb{P}^{2}}(1)$.

Corollary 2.4. We have $g^{*} \mathcal{C}^{\prime} \subset \mathcal{C}^{\prime}$ and $h^{*} \mathcal{C}^{\prime} \subset \mathcal{C}^{\prime}$.

Proof. By linearity, it suffices to consider the pullback of a prime divisor $E \subset X_{\pi}$ with $\operatorname{trop}(E) \in N^{\prime}$. If $\pi^{\prime}$ is a blowup of $\mathbb{P}^{2}$ such that $g_{\pi \pi^{\prime}}: X_{\pi^{\prime--}} X_{\pi}$ is a morphism, then $g^{*} E$ is determined in $X_{\pi}^{\prime}$ by $g_{\pi \pi^{\prime}}^{*} E$. Further, every irreducible component $E^{\prime}$ of $g_{\pi \pi^{\prime}}^{*} E$ satisfies $g_{\pi \pi^{\prime}}\left(E^{\prime}\right) \subset E$. Thus, as elements of $\mathcal{P}$, either $g\left(E^{\prime}\right)=E$ or the center of $g\left(E^{\prime}\right)$ on $X_{\pi}$ is a point in $E$. In the second case, Proposition 1.4 implies that $g\left(E^{\prime}\right)$ is non-toric with $\operatorname{trop}\left(g\left(E^{\prime}\right)\right) \in N^{\prime}$. Hence, in either case, Corollary 2.3 yields $E^{\prime} \in \mathcal{P}^{\prime}$ and therefore $g_{\pi \pi^{\prime}}^{*} E \in \mathcal{C}^{\prime}$. The proof that $h^{*} \mathcal{C}^{\prime} \subset \mathcal{C}^{\prime}$ is identical. 
Next, we study the action of $g$ on toric b-divisor classes.

Lemma 2.5. We have $g^{*} H=2 H+R$, where $R \in \mathcal{C}$ satisfies $h^{*} R \in \mathcal{C}^{\prime}$.

Proof. We use the notation introduced earlier in the subsection. On $X^{0}, H$ is represented by the divisor $\frac{1}{3} \sum_{j=0}^{2}\left(L_{j}+2 F_{j}\right)$, so $g^{*} H$ is represented by

$$
\frac{1}{3} \sum_{j=0}^{2}\left(2 L_{j}+F_{j}\right)=2 H+R,
$$

where $R:=-\sum_{j=0}^{2} F_{j}$. It only remains to see that $h^{*} R \in \mathcal{C}^{\prime}$. Pick a blowup $\pi: X_{\pi} \rightarrow \mathbb{P}^{2}$ such that $h$ induces a morphism $h_{\pi}: X_{\pi} \rightarrow X^{0}$. Then, $h^{*} R$ is represented by the divisor $\sum_{j=0}^{2} h_{\pi}^{*} F_{j}$ on $X_{\pi}$. Every irreducible component $F \subset X_{\pi}$ of $h_{\pi}^{*} F_{j}$ satisfies $h_{\pi}(F) \subset F_{j}$. Applying Proposition 1.4 if $h_{\pi}(F)$ is a point, we find that the prime $h(F) \in \mathcal{P}$ is nontoric, and $\operatorname{trop}(h(F))$ is commensurate with $-1-i, 1$ or $i$. Then, Proposition 1.5 implies that $F$ is a non-toric prime with $\zeta \operatorname{trop}(h(F))$ commensurate with $-1-i, 1$ or $i$. We conclude that $F \in \mathcal{P}^{\prime}$, and $h^{*} F_{j} \in \mathcal{C}^{\prime}$.

Lemma 2.6. If $A \in \mathcal{C}^{\text {tor }}$ and $(A \cdot H)=0$, then $g^{*} A=A$.

Proof. There exists a toric blowup $\pi: X_{\pi} \rightarrow \mathbb{P}^{2}$ such that $A$ is represented by a torusinvariant $\pi$-exceptional divisor on $X_{\pi}$. Let $\mu: X_{\pi}^{0} \rightarrow X_{\pi}$ be the blowup of $X_{\pi}$ at $p_{0}, p_{1}$ and $p_{2}$. Since no irreducible component of $A$ in $X_{\pi}$ is the proper transform of one of the coordinate lines $H_{j}$, it follows that $\mu^{*} A$ is still supported on toric primes in $X_{\pi}^{0}$. By Lemma 2.1, the birational map $g_{\pi \pi}^{0}: X_{\pi}^{0}-\rightarrow X_{\pi}^{0}$ is a morphism, and $\left(g_{\pi \pi}^{0}\right)^{*} \mu^{*} A=\mu^{*} A$ in $\operatorname{Pic}\left(X_{\pi}^{0}\right)$. This implies that $g^{*} A=A$ in $\mathcal{C}$.

\subsection{Degree sequence}

Let $g$ be the involution above, $h=h_{\zeta}$ be the monomial map associated with a Gaussian integer $\zeta$ such that $\zeta^{n} \notin \mathbb{R}$ for all $n \geqslant 1$, and set

$$
f:=g \circ h .
$$

Write

$$
d_{n}=\operatorname{deg}\left(h^{n}\right)=\left(h^{n *} H \cdot H\right) \quad \text { and } \quad e_{n}=\operatorname{deg}\left(f^{n}\right)=\left(f^{n *} H \cdot H\right)
$$

for $n \geqslant 0$. In particular, $d_{0}=e_{0}=1$. Our aim is to prove the following recursion formula.

Proposition 2.7. We have

$$
e_{n}=d_{n}+\sum_{j=0}^{n-1} e_{j} d_{n-j}
$$

for $n \geqslant 0$. 
Proof. We will prove the following more precise result by induction on $n$ :

$$
\begin{aligned}
f^{n *} H & =h^{n *} H+\sum_{j=0}^{n-1} e_{j} h^{(n-j) *} H \quad \bmod \mathcal{C}^{\prime}, \\
g^{*} f^{n *} H & =h^{n *} H+\sum_{j=0}^{n} e_{j} h^{(n-j) *} H+e_{n} R \quad \bmod \mathcal{C}^{\prime} .
\end{aligned}
$$

Pairing $\left(A_{n}\right)$ with $H$ implies the desired result, since $\mathcal{C}^{\prime}$ is orthogonal to $H$.

Now, $\left(A_{0}\right)$ is trivial, and $\left(B_{n}\right)$ implies $\left(A_{n+1}\right)$ for $n \geqslant 0$, as is seen by applying $h^{*}$ and using that $h^{*} R \in \mathcal{C}^{\prime}$. It therefore suffices to prove that $\left(A_{n}\right)$ implies $\left(B_{n}\right)$ for $n \geqslant 0$.

To this end, we rewrite $\left(A_{n}\right)$ as

$$
f^{n *} H=e_{n} H+\left(h^{n *} H+\sum_{j=0}^{n-1} e_{j} h^{(n-j) *} H-e_{n} H\right) \quad \bmod \mathcal{C}^{\prime} .
$$

The expression in parentheses lies in $\mathcal{C}^{\text {tor }}$ and is orthogonal to $H$. Lemmas 2.5 and 2.6 therefore give

$$
\begin{aligned}
g^{*} f^{n *} H & =2 e_{n} H+e_{n} R+\left(h^{n *} H+\sum_{j=0}^{n-1} e_{j} h^{(n-j) *} H-e_{n} H\right) \bmod \mathcal{C}^{\prime} \\
& =h^{n *} H+\sum_{j=0}^{n} e_{j} h^{(n-j) *} H+e_{n} R \bmod \mathcal{C}^{\prime}
\end{aligned}
$$

which completes the proof.

\subsection{Dynamical degree}

Set

$$
\Delta_{h}(z):=\sum_{j=1}^{\infty} d_{j} z^{j} \quad \text { and } \quad \Delta_{f}(z):=\sum_{j=1}^{\infty} e_{j} z^{j} .
$$

These are power series with radii of convergence equal to $|\zeta|^{-1}$ and $\lambda^{-1}$, respectively, where $\lambda$ is the dynamical degree of $f$. Proposition 2.7 shows that

$$
\left(2+\Delta_{f}(z)\right)\left(1-\Delta_{h}(z)\right)=2
$$

for $|z|<\min \left\{\lambda^{-1},|\zeta|^{-1}\right\}$.

Proposition 2.8. The dynamical degree $\lambda=\lambda(f)$ satisfies $\lambda>|\zeta|$, and $\lambda$ is the unique positive solution to the equation $\sum_{j=1}^{\infty} d_{j} \lambda^{-j}=1$, where $d_{j}=\operatorname{deg} h^{j}$. 
Proof. By submultiplicativity, we have

$$
|\zeta|=\lim _{j \rightarrow \infty} d_{j}^{1 / j}=\inf _{j} d_{j}^{1 / j}
$$

hence $d_{j} \geqslant|\zeta|^{j}$ for all $j$. Thus, $\Delta_{h}(t)$ is positive and strictly increases from 0 to $\infty$ on the interval $\left(0,|\zeta|^{-1}\right)$. Similarly, $\Delta_{f}(t)$ increases from 0 to $\infty$ on $\left(0,|\lambda|^{-1}\right)$. The equation

$$
2+\Delta_{f}(t)=\frac{2}{1-\Delta_{h}(t)}
$$

therefore implies that $t=|\lambda|^{-1}$ is the unique element of $\left(0,|\zeta|^{-1}\right)$ for which $\Delta_{h}(t)=1$.

Now, recall from $\S 1.3$ that $d_{j}=\psi\left(\zeta^{j}\right)$, where $\psi$ is a convex, non-negative and piecewise $\mathbb{R}$-linear function on $\mathbb{C}$ given by equation (1.1) and illustrated in Figure 1 . Set

$$
\alpha=\lambda^{-1} \zeta
$$

Then $|\alpha|<1$, and $\alpha$ is a solution to the equation

$$
1=\operatorname{Re} \Phi(\alpha)
$$

where $\Phi$ is a complex analytic function on the unit disk given by

$$
\Phi(z):=\sum_{j=1}^{\infty} \gamma(j) z^{j}
$$

and where the coefficient $\gamma(j)$ is the unique element $\gamma \in \Gamma_{0}$ for which $\operatorname{Re}\left(\gamma \alpha^{j}\right)$, or equivalently $\operatorname{Re}\left(\gamma \zeta^{j}\right)$, is maximized; see Figure 1. If we write

$$
\theta=\frac{1}{2 \pi} \arg (\alpha)=\frac{1}{2 \pi} \arg (\zeta) \in(0,1) \backslash \mathbb{Q}
$$

it follows that $\gamma(j)$ only depends on the image of $j \theta$ in $\mathbb{R} \bmod \mathbb{Z}$, and more specifically which interval $\left(\frac{1}{8} k, \frac{1}{8}(k+1)\right)$ contains $j \theta \bmod 1$.

\section{Proof of transcendence}

We will spend the remainder of this article proving by contradiction that the number $\alpha$ in equation (2.3), and therefore the dynamical degree $\lambda(f)$, is transcendental. All that really matters going forward is that $|\alpha|<1$, that $\theta=\arg \alpha / 2 \pi$ is irrational, and that $\operatorname{Re} \Phi(\alpha)=1$, where $\Phi(z)$ is given by equation (2.5). Our arguments will be purely number theoretic, making no further use of algebraic geometry or dynamics. 


\subsection{Setup}

Since $\theta \notin \mathbb{Q}$, the sequence $(\gamma(j))_{j \geqslant 1}$ is aperiodic. Nevertheless, as we will make precise below, it comes close to being $n$-periodic when $n$ is chosen to be the denominator in some continued fraction approximant $m / n$ of $\theta$. For such $n$, it will be illuminating to compare the analytic function $\Phi(z)$ with approximations by rational functions of the form

$$
\Phi_{n}(z):=\left(1-z^{n}\right)^{-1} \sum_{1 \leqslant j \leqslant n} \gamma(j) z^{j}=\sum_{j \geqslant 1} \gamma_{n}(j) z^{j},
$$

where $\gamma_{n}(j)$ denotes the $n$-periodic extension of the initial sequence $\gamma(1), \ldots, \gamma(n)$.

Lemma 3.1. For any sufficiently large $n \in \mathbb{N}$, we have $0<\operatorname{Re} \Phi_{n}(\alpha)<1$.

Proof. By definition, we have

$$
1-\operatorname{Re} \Phi_{n}(\alpha)=\operatorname{Re}\left(\Phi(\alpha)-\Phi_{n}(\alpha)\right)=\sum_{j>n} \operatorname{Re}\left(\left(\gamma(j)-\gamma_{n}(j)\right) \alpha^{j}\right) .
$$

Since $|\alpha|<1$ and $\Gamma_{0}=\{-2, \pm 2 i, 1 \pm 2 i\}$ is finite, the right-hand side tends to zero as $n \rightarrow \infty$; in particular, $\operatorname{Re} \Phi_{n}(\alpha)>0$ for large $n$. Now, for each $j, \gamma(j)$ maximizes $\operatorname{Re}\left(\gamma \alpha^{j}\right)$ over $\gamma \in \Gamma_{0}$, so $\operatorname{Re}\left(\left(\gamma(j)-\gamma_{n}(j)\right) \alpha^{j}\right) \geqslant 0$. Thus, $\operatorname{Re} \Phi_{n}(\alpha) \leqslant 1$, and to see that the inequality is strict, it suffices to find a single $j$ such that $\operatorname{Re}\left(\left(\gamma(j)-\gamma_{n}(j)\right) \alpha^{j}\right) \neq 0$. Since $\theta \notin \mathbb{Q}$, we can find $p \geqslant 1$ such that $p \theta \in\left(\frac{7}{8}, 1\right) \bmod 1$. Assume $n>p$, and pick $m \geqslant 1$ such that, if $j=m n+p$, then $j \theta \in\left(0, \frac{1}{8}\right) \bmod 1$. Then, from Figure 1, we see that

$$
\gamma(j)-\gamma_{n}(j)=\gamma(j)-\gamma(p)=(1-2 i)-(1+2 i)=-4 i .
$$

Since $\arg \left(\alpha^{j}\right) \in\left(0, \frac{1}{4} \pi\right)$, it follows that $\operatorname{Re}\left(-4 i \alpha^{j}\right)>0$.

Lemma 3.1 tells us that $0<\operatorname{Re}\left(\Phi(\alpha)-\Phi_{n}(\alpha)\right)<1$ for large $n$. To obtain better bounds, we clear the denominator in the definition of $\Phi_{n}(z)$, setting

$$
\begin{aligned}
\Psi_{n}(z): & =2\left|1-z^{n}\right|^{2} \operatorname{Re}\left(\Phi(z)-\Phi_{n}(z)\right) \\
& =2 \operatorname{Re}\left(\left(1-\bar{z}^{n}\right)\left(\sum_{j=1}^{\infty}\left(1-z^{n}\right) \gamma(j) z^{j}-\sum_{j=1}^{n} \gamma(j) z^{j}\right)\right) \\
& =2 \operatorname{Re}\left(\left(1-\bar{z}^{n}\right)\left(\sum_{j=n+1}^{\infty} \gamma(j) z^{j}-z^{n} \sum_{j=1}^{\infty} \gamma(j) z^{j}\right)\right) \\
& =2 \operatorname{Re}\left(\left(1-\bar{z}^{n}\right) \sum_{j=n+1}^{\infty}(\gamma(j)-\gamma(j-n)) z^{j}\right) .
\end{aligned}
$$

Since $|\alpha|<1$, we have that $0<\Psi_{n}(\alpha)<1$ for large $n$. The final expression for $\Psi_{n}$ makes the following terminology convenient. 
Definition 3.2. We say that an index $j>n$ is $n$-regular if $\gamma(j)=\gamma(j-n)$, and that it is $n$-irregular otherwise.

Since $\theta$ is irrational, there are infinitely many $n$-irregular indices, but they nevertheless form a rather sparse subset of $\mathbb{N}$, as will be explored below. Our arguments will depend on how well $\theta$ can be approximated by rational numbers. Recall (from e.g. [HW, Chapters $\mathrm{X}$ and XI]) that any irrational number $t \in \mathbb{R}$ admits an infinite sequence of continued fraction approximants $m_{j} / n_{j}$, with $n_{j}$ strictly increasing, $m_{j}$ coprime to $n_{j}$, and $\left|n_{j} t-m_{j}\right|<1 / n_{j}$ for all $j \in \mathbb{N}$.

Proposition 3.3. Let $t \in \mathbb{R}$ be an irrational number with continued fraction approximants $m_{j} / n_{j}, j \in \mathbb{N}$. Then, the following are equivalent:

(i) there exists $\kappa>0$ such that $\left|n_{j} t-m_{j}\right| \geqslant \kappa / n_{j}$ for all $j \in \mathbb{N}$;

(ii) there exists $\kappa>0$ such that $|n t-m| \geqslant \kappa / n$ for all $m, n \in \mathbb{Z}$ with $n>0$;

(iii) there exists $A$ such that $n_{j+1} \leqslant A n_{j}$ for all $j \in \mathbb{N}$;

(iv) the coefficients in the continued fraction expansion of $t$ are uniformly bounded.

Proof. Suppose first that (i) holds. As a consequence of [Bug, Corollary 1.4], for each $j$ we have $\left|n_{j} t-m_{j}\right|<1 / n_{j+1}$, and hence $\kappa / n_{j}<1 / n_{j+1}$, which gives that (iii) holds with $A=\kappa^{-1}$. Next, suppose that (iii) holds. If $a_{k}$ is the $k$ th coefficient in the continued fraction of $t$, then $n_{j+1}=a_{j+1} n_{j}+n_{j-1}$ for $j \geqslant 2$ [Bug, Theorem 1.3], and so $a_{j+1} \leqslant A$ for all $j \geqslant 2$, which gives (iv). Finally, Bugeaud [Bug, Theorem 1.9 and Definition 1.3] gives that (iv) implies (ii), and it is immediate that (ii) implies (i). This completes the proof.

We follow common convention, saying that $t$ is badly approximable if it satisfies conditions (i)-(iv) in Proposition 3.3. Because of (iv), which we do not directly use here, badly approximable $t$ are sometimes called irrational numbers of bounded type.

Our proof that $\alpha$ is transcendental is substantially simpler if $\theta$ is well (i.e. not badly) approximable. Since the set of all badly approximable numbers is small, having e.g. zero Lebesgue measure in $\mathbb{R}[\mathrm{HW}$, Theorem 196], it is reasonable to pose the following.

Question 3.4. Does there exist a Gaussian integer $\zeta$ with argument $2 \pi \theta$ for $\theta \in \mathbb{R}$ irrational and well approximable?

Unfortunately, the answer is not (as far as we are aware) presently known. So, our arguments will deal with the possibility that $\theta$ is badly approximable, too.

\subsection{A theorem of Evertse}

We now introduce one of our two main technical tools for estimating $\Psi_{n}(\alpha)$. Let $K$ be a number field of degree $d:=[K: \mathbb{Q}]$. Let $M(K)$ denote the set of places of $K$. Recall (from 
e.g. [EG]) that $M(K)$ is the disjoint union of the set $M_{\mathrm{inf}}(K)$ of infinite places and the set $M_{\text {fin }}(K)$ of finite places of $K$. A place $v \in M(K)$ determines a normalized absolute value $|\cdot|_{v}: K \rightarrow[0, \infty)$ as follows.

If $v \in M_{\text {fin }}(K)$ is finite, corresponding to a prime ideal $\mathfrak{p}$ of the ring of integers $\mathcal{O}_{K}$ of $K$, then the order $\operatorname{ord}_{\mathfrak{p}} x$ of $x \in \mathcal{O}_{K}$ is the largest power $m \geqslant 0$ such that $x \in \mathfrak{p}^{m}$. For general $x \in K^{\times}$, one sets

$$
\operatorname{ord}_{\mathfrak{p}} x:=\operatorname{ord}_{\mathfrak{p}} a-\operatorname{ord}_{\mathfrak{p}} b
$$

where $a, b \in \mathcal{O}_{K}$ satisfy $x=a / b$. Then,

$$
|x|_{v}:= \begin{cases}0, & \text { if } x=0 \\ \mathrm{~N}(\mathfrak{p})^{-\operatorname{ord}_{\mathfrak{p}}(x)}, & \text { if } x \neq 0,\end{cases}
$$

where $\mathrm{N}(\mathfrak{p})$ is the cardinality of the finite field $\mathcal{O}_{K} / \mathfrak{p}$. If $v \in M_{\mathrm{inf}}(K)$ is an infinite place, then $v$ is either real or complex. In the first case, $v$ corresponds to a real embedding $\tau: K \rightarrow \mathbb{R}$, and we take $|x|_{v}=|\tau(x)|$, where $|\cdot|$ is the ordinary absolute value on $\mathbb{R}$. In the second case, $v$ corresponds to a conjugate pair $\tau, \bar{\tau}: K \rightarrow \mathbb{C}$ of complex embeddings, and we take

$$
|x|_{v}=|\tau(x)|^{2}=|\bar{\tau}(x)|^{2} .
$$

A non-zero element $x \in K$ has the property that $|x|_{v}=1$ for all but finitely many places. With the above normalizations, the following product formula holds:

$$
\prod_{v \in M(K)}|x|_{v}=1 \quad \text { for } x \in K^{\times} .
$$

If $S \subset M(K)$ is a finite set of places containing all infinite places, then we call

$$
\mathcal{O}_{K, S}:=\left\{a \in K:|a|_{v} \leqslant 1 \text { for all } v \in M(K) \backslash S\right\}
$$

the ring of $S$-integers in $K$. Note that, if $S=M_{\mathrm{inf}}(K)$, then $\mathcal{O}_{K, S}=\mathcal{O}_{K}$ is just the usual ring of integers. Given a vector $\mathbf{x}=\left(x_{1}, \ldots, x_{m}\right) \in \mathcal{O}_{K, S}^{m}$, we set

$$
H_{S}(\mathbf{x})=\prod_{v \in S} \max \left\{\left|x_{1}\right|_{v}, \ldots,\left|x_{m}\right|_{v}\right\} .
$$

The following general result of Evertse [E] (also see [EG, Proposition 6.2.1]) on unit equations plays a central role in the sequel.

THEOREM 3.5. Let $S \subset M(K)$ be a finite set of places of $K$ containing all infinite places, $m \geqslant 2$ be an integer, and $\varepsilon>0$. There is a constant $c=c(K, S, m, \varepsilon)>0$ such that, if $\mathbf{x}=\left(x_{1}, \ldots, x_{m}\right) \in \mathcal{O}_{K, S}^{m}$ and $\sum_{k \in I} x_{k} \neq 0$ for every non-empty subset $I \subset\{1,2, \ldots, m\}$, then, for any $v_{0} \in S$,

$$
\left|x_{1}+\ldots+x_{m}\right|_{v_{0}} \geqslant c \frac{\max \left\{\left|x_{1}\right|_{v_{0}}, \ldots,\left|x_{m}\right|_{v_{0}}\right\}}{H_{S}(\mathbf{x})^{\varepsilon} \prod_{v \in S} \prod_{k=1}^{m}\left|x_{k}\right|_{v}} .
$$


We refer to any quantity of the form $\sum_{k \in I} x_{k}$, with $I \subset\{1, \ldots, m\}$ non-empty, as a non-trivial subsum of $x_{1}+\ldots+x_{m}$. The assumption that no non-trivial subsum vanishes implies among other things that $x_{k} \neq 0$ for all $k$.

\subsection{Initial choices and estimates}

From now on, we assume that $\alpha$ is an algebraic number, our final goal being to reach a contradiction. We fix the number field in the previous subsection to be a(n embedded) Galois extension $K \subset \mathbb{C}$ of $\mathbb{Q}$ that contains $\alpha, \bar{\alpha}$ and $i$. Any other embedding $K \hookrightarrow \mathbb{C}$ restricts to either the identity or $z \mapsto \bar{z}$ on $\mathbb{Q}(i)$. Hence, every infinite place $v$ of $K$ is complex, and the restriction of $|\cdot|_{v}$ to $\mathbb{Q}(i)$ is the same for all infinite places $v \in M_{\text {inf }}(K)$. We take $v_{0} \in M_{\mathrm{inf}}(K)$ to be the infinite place corresponding to the given embedding; i.e. $|a|_{v_{0}}:=|a|^{2}$, where $|\cdot|$ is the restriction to $K$ of the usual absolute value on $\mathbb{C}$.

We let $\Gamma= \pm \Gamma_{0} \cup\left(\Gamma_{0}-\Gamma_{0}\right)$. Then, $\Gamma$ contains all coefficients $\gamma(j)$ in the series defining $\Phi(z)$, as well as all differences $\gamma(j)-\gamma(j-n), j>n$. Specifically, $\Gamma$ is the set of the following 25 Gaussian integers:

$$
\Gamma:=\{0, \pm 2, \pm 2 i, \pm 1 \pm 2 i, \pm 4, \pm 4 i, \pm 2 \pm 4 i, \pm 3 \pm 2 i, \pm 1 \pm 4 i\}
$$

Note for later estimates that, if $\gamma \in \Gamma \backslash\{0\}$ and $v \in M_{\text {inf }}(K)$, then

$$
4 \leqslant|\gamma|_{v}=|\gamma|_{v_{0}}=|\gamma|^{2} \leqslant 20
$$

Finally, we fix $S \subset M(K)$ to be the set of all infinite places of $K$ together with all finite places $v$ such that $|x|_{v} \neq 1$ for some $x \in\{\alpha, \bar{\alpha}\} \cup \Gamma$.

LEMma 3.6. There is a positive constant $R$ (depending on $\Gamma$ and $\alpha$ ) such that, for any positive integer $n$ and any degree-n polynomial

$$
P(z, w)=\sum_{0 \leqslant j+k \leqslant n} \gamma_{j k} z^{j} w^{k}
$$

with coefficients $\gamma_{j k} \in \Gamma$, the quantity $x=P(\alpha, \bar{\alpha})$ satisfies

$$
\prod_{v \in S}|x|_{v} \leqslant \prod_{v \in M(K)} \max \left\{|x|_{v}, 1\right\} \leqslant R^{n} .
$$

The number $x$ in this lemma is an $S$-integer by construction. Though the polynomial $P$ used to define $x$ need not be unique, we will be somewhat imprecise and say that $x$ is a polynomial of degree $n$ in $\alpha$ and $\bar{\alpha}$ with coefficients in $\Gamma$. Whenever we apply Theorem 3.5 , it will be to a vector $\left(x_{1}, \ldots, x_{m}\right)$ whose components are all polynomials of this sort. 
Proof. Pick a positive integer $b$ such that $b \Gamma, b \alpha$ and $b \bar{\alpha}$ are all contained in $\mathcal{O}_{K}$. Then,

$$
b^{n+1} x=\sum_{0 \leqslant j+k \leqslant n}\left(b \gamma_{j k}\right)(b \alpha)^{j}(b \bar{\alpha})^{k} b^{n-j-k} \in \mathcal{O}_{K} .
$$

Thus, $\left|b^{n+1}\right|_{v} \leqslant 1$ and $\left|b^{n+1} x\right|_{v} \leqslant 1$ for every place $v \in M_{\text {fin }}(K)$, so

$$
\prod_{v \in M_{\text {fin }}(K)} \max \left\{1,|x|_{v}\right\} \leqslant \prod_{v \in M_{\text {fin }}(K)}\left|b^{-(n+1)}\right|_{v}=\prod_{v \in M_{\text {inf }}(K)}\left|b^{(n+1)}\right|_{v}=\left(b^{n+1}\right)^{d},
$$

where we used the product formula (3.2) and the fact that the degree $d$ of $K$ is twice the number of (complex) infinite places. Let $R_{0}$ be the maximum among 1 and the quantities $|\sigma(\alpha)|$, as $\sigma$ ranges over elements of the Galois group $\operatorname{Gal}(K: \mathbb{Q})$. Then, for any $v \in M_{\text {inf }}(K)$, we have

$$
|x|_{v} \leqslant(n+1)^{4} R_{0}^{2 n} \max _{\gamma \in \Gamma}|\gamma|_{v}=20(n+1)^{4} R_{0}^{2 n}
$$

and

$$
\prod_{v \in M_{\text {inf }}(K)} \max \left\{1,|x|_{v}\right\} \leqslant 20^{d / 2}(n+1)^{2 d} R_{0}^{d n} .
$$

Putting the estimates for finite and infinite places together then gives

$$
\prod_{v \in S}|x|_{v} \leqslant \prod_{v \in M(K)} \max \left\{1,|x|_{v}\right\} \leqslant\left(\sqrt{20}(n+1)^{2} R_{0}^{n}\right)^{d} b^{(n+1) d} \leqslant R^{n}
$$

for $R$ (depending on $R_{0}, b$ and $d$ ) large enough and all $n \geqslant 1$.

Corollary 3.7. If $x_{1}, \ldots, x_{m}$ are polynomials as in Lemma 3.6 and

$$
\sum_{k=1}^{m} \operatorname{deg} x_{k} \leqslant n
$$

then

$$
H_{S}\left(x_{1}, \ldots, x_{m}\right) \leqslant R^{n}
$$

Proof. Let $n_{k}=\operatorname{deg} x_{k}$. Then, by Lemma 3.6,

$$
H_{S}\left(x_{1}, \ldots, x_{m}\right)=\prod_{v \in S} \max \left\{\left|x_{1}\right|_{v}, \ldots,\left|x_{m}\right|_{v}\right\} \leqslant \prod_{v \in S} \prod_{k=1}^{m} \max \left\{1,\left|x_{k}\right|_{v}\right\} \leqslant \prod_{k=1}^{m} R^{n_{k}} \leqslant R^{n} .
$$

We conclude by noting that the left-hand estimate in Lemma 3.6 can be strengthened when $x$ is a monomial.

Lemma 3.8. If $x=\gamma \alpha^{j} \bar{\alpha}^{k}$ for some non-zero $\gamma \in \Gamma$, then $\prod_{v \in S}|x|_{v}=1$.

Proof. This follows from the product formula (3.2) and the fact that

$$
|x|_{v}=|\gamma|_{v}|\alpha|_{v}^{j}|\bar{\alpha}|_{v}^{k}=1
$$

for all places $v \notin S$. 


\subsection{The well-approximable case}

From now on, we let $m_{j} / n_{j}, j \in \mathbb{N}$ denote the continued fraction approximants of $\theta$. In this section we complete the proof that $\alpha$ is transcendental under the assumption that $\theta$ is well approximable.

Proposition 3.9. Suppose that $\theta$ is well approximable. Then, for any $C \geqslant 1$, there are arbitrarily large $n \in \mathbb{N}$ such that all indices $j \in(n, C n]$ are $n$-regular.

Proof. Let $\varepsilon=1 / 16(C+1)$. Since $\theta$ is well approximable, Proposition 3.3 (i) says that there exist infinitely many $n$ such that $|n \theta-m|<\varepsilon / n$ for some $m \in \mathbb{N}$ coprime to $n$. We claim that any such $n$ will do.

To see this, fix $j \in(n, C n]$ and let $k$ be the integer closest to $8 j \theta$. If $\left|j \theta-\frac{1}{8} k\right| \geqslant \varepsilon / n$, then either $j \theta$ and $(j-n) \theta$ are both equivalent, $\bmod 1$, to elements of $\left(\frac{1}{8}(k-1), \frac{1}{8} k\right)$, or both are equivalent to elements of $\left(\frac{1}{8} k, \frac{1}{8}(k+1)\right)$. Hence (see Figure 1) $\gamma(j \theta)=\gamma((j-n) \theta)$, i.e. $j$ is $n$-regular. If instead $\left|j \theta-\frac{1}{8} k\right|<\varepsilon / n$, then

$$
|8 m j-k n| \leqslant 8\left|j(m-n \theta)+n\left(j \theta-\frac{k}{8}\right)\right|<8 \varepsilon\left(\frac{j}{n}+1\right) \leqslant 8 \varepsilon(C+1)=\frac{1}{2} .
$$

Hence, $8 m j=k n$, and since $\operatorname{gcd}(m, n)=1$, it follows that $j=\frac{1}{8} k^{\prime} n$, where $k^{\prime}=k / m \in(8,8 C]$ is an integer. Then, we have on the one hand that

$$
j \theta-\frac{1}{8} k=\frac{1}{8} k^{\prime}(n \theta-m)
$$

but on subtracting $n \theta-m$ from both sides, we also obtain

$$
(j-n) \theta-\frac{1}{8}(k-8 m)=\frac{1}{8}\left(k^{\prime}-8\right)(n \theta-m) .
$$

Since $k^{\prime}>8$, the right-hand sides of the last two equations have the same sign; and their magnitudes are each bounded above by $\frac{1}{8} k^{\prime}|n \theta-m|=\left|j \theta-\frac{1}{8} k\right|<\frac{1}{16}$, because of our choice of $k$. So, if $n \theta-m>0$, then $j \theta \in\left(\frac{1}{8} k, \frac{1}{8}(k+1)\right)$ and $(j-n) \theta \in\left(\frac{1}{8} k-m, \frac{1}{8}(k+1)-m\right)$; and, if $n \theta-m<0$, then $j \theta \in\left(\frac{1}{8}(k-1), \frac{1}{8} k\right)$ and $(j-n) \theta \in\left(\frac{1}{8}(k-1)-m, \frac{1}{8} k-m\right)$. Either way, $\gamma(j)=\gamma(j-n)$, i.e. $j$ is $n$-regular.

Now, let $\left(x_{1}, x_{2}\right)=\left(-2\left|1-\alpha^{n}\right|^{2}, 2\left|1-\alpha^{n}\right|^{2} \operatorname{Re} \Phi_{n}(\alpha)\right)$.

COROLLARY 3.10. If $\theta$ is well approximable, then, for any $C \geqslant 1$, there are arbitrarily large $n \in \mathbb{N}$ such that

$$
\left|x_{1}+x_{2}\right| \leqslant \frac{8 \sqrt{5}|\alpha|^{C n}}{1-|\alpha|}
$$


Proof. From equation (3.1) and $\operatorname{Re} \Phi(\alpha)=1$, one sees that

$$
\left|x_{1}+x_{2}\right|=\left|\Psi_{n}(\alpha)\right| \leqslant 4\left|\sum_{j>n}(\gamma(j)-\gamma(j-n)) \alpha^{j}\right|=4\left|\sum_{j>C n}(\gamma(j)-\gamma(j-n)) \alpha^{j}\right|
$$

for any $n$ large enough that Proposition 3.9 holds. The estimate in the corollary now follows from the fact that no element of $\Gamma$ has magnitude larger than $\sqrt{20}$.

We may apply Theorem 3.5 to get a complementary bound for $\left|x_{1}+x_{2}\right|$. Take $v_{0}$ and $S$ as in the beginning of $\S 3.3$. Note that

$$
x_{1}=-2\left(1-\alpha^{n}\right)\left(1-\bar{\alpha}^{n}\right) \quad \text { and } \quad x_{2}=\left(1-\bar{\alpha}^{n}\right) \sum_{j=1}^{n} \gamma(j) \alpha^{j}+\left(1-\alpha^{n}\right) \sum_{j=1}^{n} \overline{\gamma(j)} \bar{\alpha}^{j}
$$

are polynomials in $\alpha$ and $\bar{\alpha}$ with degree $2 n$ and coefficients in $\Gamma$. Further, non-trivial subsums of $x_{1}+x_{2}$ do not vanish: $\left|x_{1}\right|>1$ for large $n$ because $|\alpha|<1$, and Lemma 3.1 tells us that $x_{2} \neq 0$ and $x_{1}+x_{2} \neq 0$ for large $n$. Hence, Theorem 3.5, together with Lemma 3.6 and Corollary 3.7 , says, for any $\varepsilon>0$, that

$$
\left|x_{1}+x_{2}\right|^{2}=\left|x_{1}+x_{2}\right|_{v_{0}} \geqslant c \frac{\max \left\{\left|x_{1}\right|^{2},\left|x_{2}\right|^{2}\right\}}{H_{S}\left(x_{1}, x_{2}\right)^{\varepsilon} \prod_{v \in S}\left|x_{1}\right|_{v}\left|x_{2}\right|_{v}} \geqslant \frac{c}{R^{4 \varepsilon n} \cdot R^{4 n}}=c R^{-4 n(1+\varepsilon)} .
$$

If $\theta$ is well approximable, then we can compare this lower bound for $\left|x_{1}+x_{2}\right|$ with the upper bound from Corollary 3.10, obtaining that

$$
|\alpha|^{C n} \geqslant c^{\prime} R^{-2 n(1+\varepsilon)}
$$

for any $C \geqslant 1, \varepsilon>0$ fixed, some constant $c^{\prime}=c^{\prime}(\varepsilon)>0$ and arbitrarily large $n \in \mathbb{N}$. Taking $\varepsilon=1$ and $C$ large enough, e.g.

$$
C=-5 \frac{\log R}{\log |\alpha|}
$$

we arrive at a contradiction. So, if $\theta$ is well approximable, then $\alpha$ is transcendental.

\subsection{Unit equations}

We need a little extra machinery from the theory of unit equations to deal with the possibility that $\theta$ is badly approximable. Specifically, we need the following result due to Evertse, Schlickewei and Schmidt; see [ESS, Theorem 1.1] and also [EG, Theorem 6.1.3]. To state the theorem, we recall that, if $a_{1}, \ldots, a_{m} \in L$ are (non-zero) elements of a field $L$, then a solution $y_{1}, \ldots, y_{m} \in L$ of

$$
a_{1} y_{1}+\ldots+a_{m} y_{m}=1
$$

is called non-degenerate if non-trivial subsums of the left-hand side do not vanish. And a multiplicative subgroup $H \subset\left(L^{\times}\right)^{m}$ is said to have $\operatorname{rank} r<\infty$, if there is a free abelian subgroup $H^{\prime}$ of rank $r$ such that $H / H^{\prime}$ is finite. 
Theorem 3.11. Let $L$ be a field of characteristic zero, let $a_{1}, \ldots, a_{m} \in L^{\times}$, and let $H \subset\left(L^{\times}\right)^{m}$ be a subgroup of finite rank. Then, there are only finitely many non-degenerate solutions $\left(y_{1}, \ldots, y_{m}\right) \in H$ of the equation $a_{1} y_{1}+\ldots+a_{m} y_{m}=1$.

Note that, while [EG, Theorem 6.1.3] is only stated for $m \geqslant 2$, it is also valid (trivially) when $m=1$. To apply the theorem, let $K$ and $\Gamma$ be as in $\S 3.3$.

LEMma 3.12. The numbers $\alpha$ and $\bar{\alpha}$ generate a free multiplicative subgroup of $\mathbb{C}^{\times}$.

Proof. We have $\arg (\alpha)=2 \pi \theta$, so if $\alpha^{j} \bar{\alpha}^{k}=1$, then $j=k$, as $\theta$ is irrational. But then $\alpha^{j} \bar{\alpha}^{k}=|\alpha|^{2 j}$, and hence $j=k=0$ since $|\alpha|<1$.

Corollary 3.13. For any integer $m \geqslant 1$, there exists $N=N(m) \in \mathbb{N}$ such that

$$
\gamma_{1} \alpha^{j_{1}} \bar{\alpha}^{k_{1}}+\ldots+\gamma_{m} \alpha^{j_{m}} \bar{\alpha}^{k_{m}} \neq 0
$$

whenever $\gamma_{1}, \ldots, \gamma_{m} \in \Gamma$ are not all zero, and $\left|j_{h}-j_{l}\right|+\left|k_{h}-k_{l}\right| \geqslant N$ for all $h \neq l$.

Proof. We may assume that $m \geqslant 2$. Since $\Gamma$ is a finite set, it suffices to consider a fixed vector $\left(\gamma_{1}, \ldots, \gamma_{m}\right)$, and we may further assume (after schrinking $m$, if necessary) that $\gamma_{k} \neq 0$ for all $k$. By Lemma 3.12, it therefore suffices to prove that, for any $\left(\gamma_{1}, \ldots, \gamma_{m}\right) \in$ $(\Gamma \backslash\{0\})^{m}$, there are only finitely many non-degenerate solutions $\left(\alpha^{j_{1}^{\prime}} \bar{\alpha}^{k_{1}^{\prime}}, \ldots, \alpha^{j_{m-1}^{\prime}} \bar{\alpha}_{m-1}^{\prime}\right)$ to the equation

$$
\gamma_{1} \alpha^{j_{1}^{\prime}} \bar{\alpha}^{k_{1}^{\prime}}+\ldots+\gamma_{m-1} \alpha^{j_{m-1}^{\prime}} \bar{\alpha}^{k_{m-1}^{\prime}}+\gamma_{m}=0
$$

This follows from Theorem 3.11 with $L=\mathbb{C}, m-1$ in place of $m, a_{k}=-\gamma_{k} / \gamma_{m}$, and $H=G^{m-1}$, where $G \subset \mathbb{C}^{\times}$is the multiplicative group generated by $\alpha$ and $\bar{\alpha}$.

Theorem 3.5 now allows us to render Corollary 3.13 effective.

Corollary 3.14. Given $\delta, \rho>0, C \geqslant 1$ and an integer $m \geqslant 1$, the following is true for $n$ large enough. Suppose $j_{1}, k_{1}, \ldots, j_{m}, k_{m} \geqslant 0$ are integers satisfying

- $j_{h}+k_{h} \leqslant C n$ for all $h$,

- $\left|j_{h}-j_{l}\right|+\left|k_{h}-k_{l}\right| \geqslant \delta n$ for all $h \neq l$, and suppose that $\gamma_{1}, \ldots, \gamma_{m} \in \Gamma$ do not all vanish. Then,

$$
\left|\gamma_{1} \alpha^{j_{1}} \bar{\alpha}^{k_{1}}+\ldots+\gamma_{m} \alpha^{j_{m}} \bar{\alpha}^{k_{m}}\right| \geqslant|\alpha|^{\min _{h: \gamma_{h} \neq 0}\left(j_{h}+k_{h}\right)+\rho n} \geqslant|\alpha|^{(C+\rho) n} .
$$

Proof. Suppose without loss of generality that no $\gamma_{k}$ vanishes. Corollary 3.13 tells us that no non-trivial subsum of the sum on the left vanishes. Let $S \subset M(K)$ and $v_{0} \in S$ be as in the beginning of $\S 3.3$, and $\left(x_{1}, \ldots, x_{m}\right)$ be the vector of monomials $x_{h}=\gamma_{h} \alpha^{j_{h}} \bar{\alpha}^{k_{h}}$. Lemma 3.8 tells us that $\prod_{v \in S} \prod_{h=1}^{m}\left|x_{h}\right|_{v}=1$. Further,

$$
\max \left\{\left|x_{1}\right|^{2}, \ldots,\left|x_{m}\right|^{2}\right\}=\max _{h}\left|\gamma_{h} \alpha^{j_{h}} \bar{\alpha}^{k_{h}}\right|^{2} \geqslant 4|\alpha|^{2 \min _{h}\left(j_{h}+k_{h}\right)},
$$


using $|\alpha|<1$ and equation (3.3), and Corollary 3.7 gives

$$
H_{S}\left(x_{1}, \ldots, x_{m}\right) \leqslant R^{C m n},
$$

for $R>0$ as in Lemma 3.6. Theorem 3.5 therefore yields

$$
\left|x_{1}+\ldots+x_{m}\right|^{2}=\left|x_{1}+\ldots+x_{m}\right|_{v_{0}} \geqslant \frac{4 c|\alpha|^{2 \min _{h}\left(j_{h}+k_{h}\right)}}{R^{C m n \varepsilon}} .
$$

Choosing $\varepsilon>0$ small enough that $R^{-C m \varepsilon}>|\alpha|^{2 \rho}$ guarantees that the first inequality of equation (3.5) holds for large $n$, completing the proof.

\subsection{The badly approximable case}

It remains to treat the case when $\theta$ is badly approximable. We recall (see [HW, Theorems 167 and 171]) that the continued fraction approximants of an irrational number alternate between over- and under-approximating, i.e. if the approximants $m_{j} / n_{j}$ of $\theta$ are indexed so that $n_{0}=1$, then we have for any odd index $j$ that

$$
\frac{m_{j-1}}{n_{j-1}}<\theta<\frac{m_{j}}{n_{j}} .
$$

The next result serves as an alternative to Proposition 3.9.

Proposition 3.15. Suppose that $\theta$ is badly approximable. Then, there exist $B>0$, $\delta>0$ and arbitrarily large $n \in \mathbb{N}$ such that the following statements hold:

(i) $j-n \geqslant \delta n$ for any $n$-irregular index $j>n$;

(ii) $\left|j-j^{\prime}\right| \geqslant \delta n$ for any distinct $n$-irregular indices $j, j^{\prime}>n$;

(iii) $\left|j-j^{\prime}-n\right| \geqslant \delta n$ for any $n$-irregular indices $j, j^{\prime}>n$ such that $j \neq j^{\prime}+n$;

(iv) for any $C \geqslant 1$, there are at most $C / \delta n$-irregular indices in the interval $(n, C n]$, and at least one n-irregular index in the interval $(C n, B C n]$.

Proof. By hypothesis (see Proposition 3.3), there exists $\kappa>0$ such that $|n \theta-m| \geqslant \kappa / n$ for any integers $m, n$ with $n>0$. In what follows, we take $m=m_{h}$ and $n=n_{h}$, with $h$ odd.

Suppose that $j>n$ is $n$-irregular, and let $k$ be the integer closest to $8 j \theta$. Since $m / n$ is a continued fraction approximant of $\theta$, we have $|n \theta-m|<1 / n$. So, one can argue as in the second paragraph of the proof of Proposition 3.9 to show that $\left|j \theta-\frac{1}{8} k\right|<1 / n$. Hence,

$$
\frac{\kappa}{8(j-n)} \leqslant|8(j-n) \theta-(k-8 m)| \leqslant 8\left|j \theta-\frac{k}{8}\right|+8|n \theta-m|<\frac{16}{n} .
$$

So, $j-n>\frac{1}{128} \kappa n$. And, if $j^{\prime}>n$ is another $n$-irregular index, then $\left|j^{\prime} \theta-\frac{1}{8} k^{\prime}\right|<1 / n$ for some $k^{\prime} \in \mathbb{N}$. Hence, if $j^{\prime} \neq j$,

$$
\frac{\kappa}{8\left|j^{\prime}-j\right|} \leqslant\left|8\left(j^{\prime}-j\right) \theta-\left(k^{\prime}-k\right)\right| \leqslant 8\left|j \theta-\frac{k}{8}\right|+8\left|j^{\prime} \theta-\frac{k^{\prime}}{8}\right|<\frac{16}{n} .
$$


So, $\left|j^{\prime}-j\right|>\frac{1}{128} \kappa n$. Similarly, if $j^{\prime}+n \neq j$, then $\left|j \theta-\frac{1}{8} k\right|,\left|j^{\prime} \theta-\frac{1}{8} k^{\prime}\right|$ and $|n \theta-m|$ are all less than $1 / n$, so now the triangle inequality gives

$$
\frac{\kappa}{8\left|j-j^{\prime}-n\right|} \leqslant\left|8\left(j-j^{\prime}-n\right) \theta-\left(k-k^{\prime}+8 m\right)\right|<\frac{24}{n},
$$

i.e. $\left|j-j^{\prime}-n\right|>\frac{1}{192} \kappa n$. All told, statements (i)-(iii) hold with $\delta=\frac{1}{192} \kappa$.

The first part of (iv) follows immediately from (ii). To prove the second part, pick $m^{\prime} / n^{\prime}=m_{h^{\prime}} / n_{h^{\prime}}$ to be the continued fraction approximant of $\theta$ with minimal even index $h^{\prime}$ such that $n_{h^{\prime}}>C n$. Since $\theta$ is badly approximable, we have $n^{\prime} \leqslant A^{2} C n$, where $A$ is the constant in the third condition of Proposition 3.3. Since $h$ is odd and $h^{\prime}>h$ is even, we also have $m^{\prime} / n^{\prime}<\theta<m / n$. Thus,

$$
0<n^{\prime} \theta-m^{\prime}<m-n \theta<\frac{1}{n},
$$

where the middle inequality comes from the fact that continued fraction approximants of $\theta$ improve as the denominators $n<n^{\prime}$ increase. Assuming $n \geqslant 8$, we infer that $n^{\prime} \theta$ is equivalent, $\bmod 1$, to an element of $\left(0, \frac{1}{8}\right)$. The inequalities above give

$$
-\frac{1}{n}<\left(n^{\prime}+n\right) \theta-\left(m^{\prime}+m\right)<0,
$$

so that $\left(n^{\prime}+n\right) \theta$ is equivalent, $\bmod 1$ to an element of $\left(\frac{7}{8}, 1\right)$. Then, $\gamma\left(n^{\prime}\right)=1-2 i$ and $\gamma\left(n+n^{\prime}\right)=1+2 i$ (see Figure 1 ), so the index $j=n^{\prime}+n$ is $n$-irregular. Since

$$
C n<j<\left(A^{2} C+1\right) n,
$$

we may take $B=A^{2}+1$ to conclude the proof.

We define $\beta_{j, k}(n) \in \Gamma$ for $j, k \in \mathbb{N}$ by (see equation (3.1))

$$
\Psi_{n}(z)=2 \operatorname{Re}\left(\left(1-\bar{z}^{n}\right) \sum_{k>n}(\gamma(k)-\gamma(k-n)) z^{k}\right)=\sum_{j+k>n} \beta_{j k}(n) z^{j} \bar{z}^{k},
$$

noting that $\beta_{j k}(n)=\overline{\beta_{k j}(n)}$ is non-zero if and only if one of the indices $j$ or $k$ is $n$ irregular (hence $>n$ ) and the other is equal to zero or $n$. Proposition 3.15 implies that, for suitable $n$, the indices of non-vanishing $\beta_{j k}(n)$ are well separated.

Corollary 3.16. Suppose that $\theta$ is badly approximable, and let $\delta>0$ be as in Proposition 3.15. Then, for every $C>\frac{1}{4} \delta$, there exists an integer $r \in[0,4 C / \delta)$ such that the following assertions hold for infinitely many $n$ :

(i) if $j, j^{\prime}, k, k^{\prime} \in \mathbb{N}$ are such that $\beta_{j k}(n) \neq 0$ and $\beta_{j^{\prime} k^{\prime}}(n) \neq 0$, then

(a) $(j, k)=\left(j^{\prime}, k^{\prime}\right)$ or $\left|j-j^{\prime}\right|+\left|k-k^{\prime}\right| \geqslant \delta n$, and

(b) $j+k=j^{\prime}+k^{\prime}$ or $\left|(j+k)-\left(j^{\prime}+k^{\prime}\right)\right| \geqslant \delta n$;

(ii) precisely $r$ of the coefficients $\beta_{j k}(n)$ with $j+k \in(n, C n]$ are non-vanishing. 
Proof. Let $B>0$ be as in Proposition 3.15, and let $Z \subset \mathbb{N}$ be an infinite subset such that all the assertions of that proposition hold for all $n \in Z$.

Then (a) follows from Proposition 3.15 (i)-(ii). Similarly, (b) follows from Proposition 3.15 (i)-(iii): indeed, we may assume, without loss of generality, that $k$ and $k^{\prime}$ are irregular, and in this case $j-j^{\prime} \in\{0, n,-n\}$.

To prove (b), set

$$
r_{n}=\#\left\{(j, k): \beta_{j, k}(n) \neq 0 \text { and } j+k \in(n, C n]\right\}
$$

for any $n \in Z$. In each pair $(j, k)$ being counted, one component is $n$-irregular and the other is equal to either zero or $n$. So, Proposition 3.15 (iv) implies that $r_{n}<4 C / \delta$ for all $n \in Z$. Hence, we can take $r=\liminf _{n \rightarrow \infty} r_{n}$ to be the smallest value of $r_{n}$ that occurs for infinitely many $n$.

Continuing to suppose that $\theta$ is badly approximable, we let $\delta>0$ be as in Proposition 3.15, and fix $C>\max \left\{1, \frac{1}{4} \delta\right\}$ (to be specified more precisely below). Let $r \geqslant 0$ and $n$ be as in Corollary 3.16. Pick $\rho \in(0, \delta)$. We will apply Theorem 3.5 to the vector

$$
\mathbf{x}=\left(x_{1}, x_{2}, \ldots, x_{r+2}\right) \in \mathcal{O}_{K, S}^{r+2},
$$

where

$$
x_{1}=-2\left|1-\alpha^{n}\right|^{2}, \quad x_{2}=2\left|1-\alpha^{n}\right|^{2} \operatorname{Re} \Phi_{n}(\alpha),
$$

and $x_{3}, \ldots, x_{r+2}$ are the non-vanishing terms $\beta_{j k}(n) \alpha^{j} \bar{\alpha}^{k}$ with $j+k \leqslant C n$ in formula (3.6) for $\Psi_{n}(\alpha)$. From equation (3.1) and $\operatorname{Re} \Phi(\alpha)=1$, we get

$$
x_{1}+x_{2}=2\left|1-\alpha^{n}\right|^{2}\left(\operatorname{Re} \Phi_{n}(\alpha)-1\right)=2\left|1-\alpha^{n}\right|^{2} \operatorname{Re}\left(\Phi_{n}(\alpha)-\Phi(\alpha)\right)=-\Psi_{n}(\alpha) .
$$

Together with equation (3.6), this gives

$$
x_{1}+\ldots+x_{r+2}=-\sum_{j+k>C n} \beta_{j k}(n) \alpha^{j} \bar{\alpha}^{k} .
$$

Let $p(n)$ denote the maximum value of $j+k$ such that $\beta_{j k}(n) \neq 0$ and $j+k \leqslant C n$. Let $q(n)$ denote the minimum value of $j+k$ such that $\beta_{j k}(n) \neq 0$ and $j+k>C n$. By Corollary $3.16(\mathrm{~b})$, we have $q(n) \geqslant p(n)+\delta n$.

Recall that, if $\beta_{j k}(n) \neq 0$, then the smaller of the indices $j$ and $k$ must equal either zero or $n$. Therefore, for fixed $n$ and $l$, there are at most four non-zero $\beta_{j k}(n)$ with $j+k=l$. So, from the previous equality, we estimate

$$
\left|\sum_{h=1}^{r+2} x_{h}\right| \leqslant \sum_{j+k>C n}\left|\beta_{j k}(n)\right||\alpha|^{j+k} \leqslant 4 \sqrt{20} \sum_{l \geqslant q(n)}|\alpha|^{l}=\frac{8 \sqrt{5}|\alpha|^{q(n)}}{1-|\alpha|}
$$

where the second inequality uses that $\beta_{j k}(n) \in \Gamma$, and hence $\left|\beta_{j k}(n)\right| \leqslant \sqrt{20}$. 
LEMMA 3.17. If $I \subset\{1,2, \ldots, r+2\}$ is non-empty, then $\sum_{h \in I} x_{h} \neq 0$.

Proof. We argue by contradiction, so suppose $\sum_{h \in I} x_{h}=0$. By Corollaries 3.13 and 3.16, we cannot have $I \subset\{3, \ldots, r+2\}$. On the other hand, for large $n,\left|x_{1}\right|>1$ because $|\alpha|<1$, and $\left|x_{2}\right|>1$ because (additionally) $\operatorname{Re} \Phi_{n}(\alpha) \rightarrow 1$. Finally, Lemma 3.1 tells us that $x_{1}+x_{2} \neq 0$ when $n$ is large. So, we cannot have $I \subset\{1,2\}$ either.

Since $\left|x_{3}+\ldots+x_{r+2}\right| \leqslant \sqrt{20} r|\alpha|^{n}<1<\left|x_{1}\right|,\left|x_{2}\right|$, both 1 and 2 belong to $I$ when $n$ is large. If the complement $J=\{1, \ldots, r+2\} \backslash I \subset\{3, \ldots, r+2\}$ is non-empty, then Corollaries 3.14 and 3.16 imply that

$$
\left|\sum_{h=1}^{r+2} x_{h}\right|=\left|\sum_{h \in J} x_{h}\right| \geqslant|\alpha|^{p(n)+\rho n}
$$

which contradicts equation (3.7) for large $n$, since $p(n) \leqslant q(n)-\delta n$ and $\rho<\delta$.

Thus, $I=\{1, \ldots, r+2\}$, which gives

$$
0=-\sum_{h=1}^{r+2} x_{h}=\sum_{j+k>C n} \beta_{j k}(n) \alpha^{j} \bar{\alpha}^{k}=\sum_{j+k=q(n)} \beta_{j k}(n) \alpha^{j} \bar{\alpha}^{k}+\sum_{j+k \geqslant q(n)+\delta n} \beta_{j k}(n) \alpha^{j} \bar{\alpha}^{k},
$$

where, in the last equality, we have used Corollary 3.16 (b). Hence, the two sums on the right-hand side have the same magnitude. Further, $q(n) \leqslant B C n$ by Proposition 3.15 (iv), so Corollary 3.14 implies that

$$
|\alpha|^{q(n)+\rho n} \leqslant\left|\sum_{j+k=q(n)} \beta_{j k}(n) \alpha^{j} \bar{\alpha}^{k}\right|=\left|\sum_{j+k \geqslant q(n)+\delta n} \beta_{j k}(n) \alpha^{j} \bar{\alpha}^{k}\right| \leqslant \frac{8 \sqrt{5}|\alpha|^{q(n)+\delta n}}{1-|\alpha|},
$$

where the first inequality follows from Corollary 3.14 and Corollary 3.16 (i), and the second inequality is obtained in the same way as equation (3.7). Since $\rho<\delta$, this is a contradiction for large $n$.

We are ready to invoke Theorem 3.5 one last time, with $S \subset M(K)$ and $v_{0} \in S$, as in the beginning of $\S 3.3$. From Lemma 3.8, and then Lemma 3.6, we obtain

$$
\prod_{v \in S}\left|x_{1}\right|_{v} \ldots\left|x_{r+2}\right|_{v}=\prod_{v \in S}\left|x_{1}\right|_{v}\left|x_{2}\right|_{v} \leqslant R^{4 n}
$$

since $x_{3}, \ldots, x_{r+2}$ are monomials in $\alpha$ and $\bar{\alpha}$, and $x_{1}$ and $x_{2}$ are polynomials of degree $2 n$; see equation (3.4). Further, $x_{3}, \ldots, x_{r+2}$ have degree at most $C n$, so Corollary 3.7 gives

$$
H_{S}(\mathbf{x}) \leqslant R^{(C r+4) n}
$$


Lemma 3.17 says that non-trivial subsums of $x_{1}+\ldots+x_{r+2}$ do not vanish. So, for fixed $\varepsilon>0$, Theorem 3.5 yields

$$
\left|x_{1}+\ldots+x_{r+2}\right|^{2} \geqslant c \frac{\max \left\{\left|x_{1}\right|^{2}, \ldots,\left|x_{r+2}\right|^{2}\right\}}{H_{S}(\mathbf{x})^{\varepsilon} \prod_{v \in S} \prod_{h=1}^{r+2}\left|x_{h}\right|_{v}} \geqslant \frac{c}{R^{(C r+4) n \varepsilon} \cdot R^{4 n}}=c R^{-(4+(C r+4) \varepsilon) n}
$$

for large $n$, since $\left|x_{1}\right| \geqslant 1$. Using the bound in the other direction from equation (3.7), we infer that, if $n \in \mathbb{N}$ is as in Corollary 3.16 and is large enough, then

$$
|\alpha|^{2 C n} \geqslant|\alpha|^{2 q(n)} \geqslant c^{\prime} R^{-(4+(C r+4) \varepsilon) n}
$$

for some constant $c^{\prime}=c^{\prime}(C, \varepsilon)>0$. So, if above we fix $C>1$ such that $|\alpha|^{C} \leqslant R^{-3}$, and then set $\varepsilon=1 /(C r+4)$, we obtain $R^{-6 n} \geqslant c^{\prime} R^{-5 n}$ for arbitrarily large $n$, which is a contradiction. We conclude that, if $\theta$ is badly approximable, $\alpha$ is transcendental.

This completes the proof of the main theorem in the introduction.

\section{References}

[AB1] Adamczewsin, B. \& Bugeaud, Y., On the complexity of algebraic numbers. I. Expansions in integer bases. Ann. of Math., 165 (2007), 547-565.

[AB2] - Dynamics for $\beta$-shifts and Diophantine approximation. Ergodic Theory Dynam. Systems, 27 (2007), 1695-1711.

[AC1] Adamczewski, B. \& Cassaigne, J., On the transcendence of real numbers with a regular expansion. J. Number Theory, 103 (2003), 27-37.

[AC2] - Diophantine properties of real numbers generated by finite automata. Compos. Math., 142 (2006), 1351-1372.

[BK1] Bedford, E. \& Kim, K., Periodicities in linear fractional recurrences: degree growth of birational surface maps. Michigan Math. J., 54 (2006), 647-670.

[BK2] - Linear recurrences in the degree sequences of monomial mappings. Ergodic Theory Dynam. Systems, 28 (2008), 1369-1375.

[BBC] Bell, J. P., Bugeaud, Y. \& Coons, M., Diophantine approximation of Mahler numbers. Proc. Lond. Math. Soc., 110 (2015), 1157-1206.

[BV] Bellon, M. P. \& Viallet, C. M., Algebraic entropy. Comm. Math. Phys., 204 (1999), 425-437.

[BiJ+] Benedetto, R., Ingram, P., Jones, R., Manes, M., Silverman, J. H. \& Tucker, T. J., Current trends and open problems in arithmetic dynamics. Bull. Amer. Math. Soc., 56 (2019), 611-685.

[Beu] Beukers, F., A refined version of the Siegel-Shidlovskii theorem. Ann. of Math., 163 (2006), 369-379.

[Bla] Blanc, J., Symplectic birational transformations of the plane. Osaka J. Math., 50 (2013), 573-590.

[BC] Blanc, J. \& CAntat, S., Dynamical degrees of birational transformations of projective surfaces. J. Amer. Math. Soc., 29 (2016), 415-471.

[BF] Bonifant, A. M. \& FornÆss, J. E., Growth of degree for iterates of rational maps in several variables. Indiana Univ. Math. J., 49 (2000), 751-778. 
[BFJ] Boucksom, S., Favre, C. \& Jonsson, M., Degree growth of meromorphic surface maps. Duke Math. J., 141 (2008), 519-538.

[Bug] Bugeaud, Y., Approximation by Algebraic Numbers. Cambridge Tracts in Mathematics, 160. Cambridge Univ. Press, Cambridge, 2004.

[Cal] Calcut, J. S., Gaussian integers and arctangent identities for $\pi$. Amer. Math. Monthly, 116 (2009), 515-530.

[Can] Cantat, S., Sur les groupes de transformations birationnelles des surfaces. Ann. of Math., 174 (2011), 299-340.

[CX] Cantat, S. \& XIE, J., On degrees of birational mappings. Math. Res. Lett., 27 (2020), 319-337.

[CZ] Corvaja, P. \& Zannier, U., Some new applications of the subspace theorem. Compositio Math., 131 (2002), 319-340.

[D] Dang, N.-B., Degrees of iterates of rational maps on normal projective varieties. Proc. Lond. Math. Soc., 121 (2020), 1268-1310.

[DF] DAng, N.-B. \& FAVRe, C., Spectral interpretations of dynamical degrees and applications. Preprint, 2020. arXiv: 2006.10262 [math.AG].

[DDG1] Diller, J., Dujardin, R. \& Guedj, V., Dynamics of meromorphic maps with small topological degree I: From cohomology to currents. Indiana Univ. Math. J., 59 (2010), 521-561.

[DDG2] - Dynamics of meromorphic mappings with small topological degree II: Energy and invariant measure. Comment. Math. Helv., 86 (2011), 277-316.

[DDG3] - Dynamics of meromorphic maps with small topological degree III: Geometric currents and ergodic theory. Ann. Sci. Éc. Norm. Supér., 43 (2010), 235-278.

[DF] Diller, J. \& Favre, C., Dynamics of bimeromorphic maps of surfaces. Amer. J. Math., 123 (2001), 1135-1169.

[DL] Diller, J. \& Lin, J.-L., Rational surface maps with invariant meromorphic two-forms. Math. Ann., 364 (2016), 313-352.

[DS1] Dinh, T.-C. \& Sibony, N., Une borne supérieure pour l'entropie topologique d'une application rationnelle. Ann. of Math., 161 (2005), 1637-1644.

[DS2] - Equidistribution problems in complex dynamics of higher dimension. Internat. J. Math., 28 (2017), 1750057, 31 pp.

[E] Evertse, J.-H., On sums of $S$-units and linear recurrences. Compositio Math., 53 (1984), 225-244.

[EG] Evertse, J.-H. \& GYőRY, K., Unit Equations in Diophantine Number Theory. Cambridge Studies in Advanced Mathematics, 146. Cambridge Univ. Press, Cambridge, 2015 .

[ESS] Evertse, J.-H., Schlickewei, H. P. \& Schmidt, W. M., Linear equations in variables which lie in a multiplicative group. Ann. of Math., 155 (2002), 807-836.

[Fa] Favre, C., Les applications monomiales en deux dimensions. Michigan Math. J., 51 (2003), 467-475.

[FJ1] Favre, C. \& Jonsson, M., Eigenvaluations. Ann. Sci. École Norm. Sup., 40 (2007), 309-349.

[FJ2] - Dynamical compactifications of $\mathbf{C}^{2}$. Ann. of Math., 173 (2011), 211-248.

[FM] Ferenczi, S. \& Mauduit, C., Transcendence of numbers with a low complexity expansion. J. Number Theory, 67 (1997), 146-161.

[Fr] Friedland, S., Entropy of polynomial and rational maps. Ann. of Math., 133 (1991), 359-368.

[Gr] Gromov, M., On the entropy of holomorphic maps. Enseign. Math., 49 (2003), 217-235. 
[Gu1] Guedj, V., Ergodic properties of rational mappings with large topological degree. Ann. of Math., 161 (2005), 1589-1607.

[Gu2] - Propriétés ergodiques des applications rationnelles, in Quelques aspects des systèmes dynamiques polynomiaux, Panor. Synthèses, 30, pp. 97-202. Soc. Math. France, Paris, 2010.

[HW] Hardy, G. H. \& Wright, E. M., An Introduction to the Theory of Numbers. Oxford Univ. Press, Oxford, 2008.

[HP] Hasselblatt, B. \& Propp, J., Degree-growth of monomial maps. Ergodic Theory Dynam. Systems, 27 (2007), 1375-1397. Correction in 27 (2007), 1999.

[JR] Jonsson, M. \& Reschke, P., On the complex dynamics of birational surface maps defined over number fields. J. Reine Angew. Math., 744 (2018), 275-297.

[KS] Kawaguchi, S. \& Silverman, J.H., On the dynamical and arithmetic degrees of rational self-maps of algebraic varieties. J. Reine Angew. Math., 713 (2016), 21-48. Correction in 761 (2020), 291-292.

[LS] Lesieutre, J. \& Satriano, M., A rational map with infinitely many points of distinct arithmetic degrees. Ergodic Theory Dynam. Systems, 40 (2020), 3051-3055.

[Man] Manin, Y. I. \& Hazewinkel, M., Cubic Forms: Algebra, Geometry, Arithmetic. NorthHolland Mathematical Library, 4. North-Holland, Amsterdam-London; American Elsevier, New York, 1974.

[Mat] Matsuzawa, Y., On upper bounds of arithmetic degrees. Amer. J. Math., 142 (2020), $1797-1820$

[McM] McMullen, C. T., Dynamics on blowups of the projective plane. Publ. Math. Inst. Hautes Études Sci., 105 (2007), 49-89.

[N] NishiokA, K., Mahler Functions and Transcendence. Lecture Notes in Mathematics, 1631. Springer, Berlin-Heidelberg, 1996.

[RS] Russakovskil , A. \& Shiffman, B., Value distribution for sequences of rational mappings and complex dynamics. Indiana Univ. Math. J., 46 (1997), 897-932.

[Sch] SchlickeweI, H.P., The $p$-adic Thue-Siegel-Roth-Schmidt theorem. Arch. Math. (Basel), 29 (1977), 267-270.

[Sha] Shafarevich, I. R., Basic Algebraic Geometry. 1. Varieties in Projective Space. Springer, Berlin-Heidelberg, 2013.

[Sib] Sibony, N., Dynamique des applications rationnelles de $\mathbf{P}^{k}$, in Dynamique et géométrie complexes (Lyon, 1997), Panor. Synthèses, 8, pp. 97-185. Soc. Math. France, Paris, 1999.

[Sil] Silverman, J.H., Dynamical degree, arithmetic entropy, and canonical heights for dominant rational self-maps of projective space. Ergodic Theory Dynam. Systems, 34 (2014), 647-678.

[T] Truong, T. T., Relative dynamical degrees of correspondences over a field of arbitrary characteristic. J. Reine Angew. Math., 758 (2020), 139-182.

[Ue] Uehara, T., Rational surface automorphisms with positive entropy. Ann. Inst. Fourier (Grenoble), 66 (2016), 377-432.

[Ur] URECH, C., Remarks on the degree growth of birational transformations. Math. Res. Lett., 25 (2018), 291-308.

[V] Viallet, C.-M., Algebraic dynamics and algebraic entropy. Int. J. Geom. Methods Mod. Phys., 5 (2008), 1373-1391. 
JASON P. BELL

Department of Pure Mathematics

University of Waterloo

Waterloo, ON N2L 3G1

Canada

jpbell@uwaterloo.ca

MATtias Jonsson

Department of Mathematics

University of Michigan

Ann Arbor, MI 48109-1043

U.S.A.

mattiasj@umich.edu

Received November 14, 2019

Received in revised form November 29, 2020
JEFFrey Diller

Department of Mathematics

University of Notre Dame

Notre Dame, IN 46556

U.S.A.

diller.1@nd.edu 